\title{
Mechanisms of airfoil noise near stall conditions
}

\author{
Giovanni Lacagnina $\oplus^{*}$ and Paruchuri Chaitanya \\ Institute of Sound and Vibration Research, University of Southampton, \\ Southampton SO17 1BJ, United Kingdom
}

Tim Berk

Aerodynamics and Flight Mechanics Research Group, University of Southampton, Southampton SO17 1BJ, United Kingdom

Jung-Hoon Kim

Faculty of Engineering, University of Nottingham, Nottingham NG7 2RD, United Kingdom

Phillip Joseph

Institute of Sound and Vibration Research, University of Southampton, Southampton SO17 1BJ, United Kingdom

Bharathram Ganapathisubramani

Aerodynamics and Flight Mechanics Research Group, University of Southampton,

Southampton SO17 1BJ, United Kingdom

Seyed Mohammad Hasheminejad

Department of Mechanical, Aerospace and Civil Engineering, Brunel University, London UB8 3PH, United Kingdom

Tze Pei Chong

Department of Mechanical, Aerospace and Civil Engineering, Brunel University, London UB8 3PH, United Kingdom

Oksana Stalnov

Technion-Israel Institute of Technology, Haifa 3200003, Israel

Kwing-So Choi

Faculty of Engineering, University of Nottingham, Nottingham NG7 2RD, United Kingdom

Muhammad Farrukh Shahab, Mohammad Omidyeganeh, and Alfredo Pinelli

School of Mathematics, Computer Science and Engineering, City University London, London EC1V OHB, United Kingdom

\section{(Received 11 March 2019; published 6 December 2019)}

The focus of this paper is on investigating the noise produced by an airfoil at high angles of attack over a range of Reynolds number $\operatorname{Re} \approx 2 \times 10^{5}-4 \times 10^{5}$. The objective is not modeling this source of noise but rather understanding the mechanisms of generation for surface pressure fluctuations, due to a separated boundary layer, that are then scattered by the trailing edge. To this aim, we use simultaneous noise and surface pressure

\footnotetext{
*g.lacagnina@soton.ac.uk
} 
measurement in addition to velocimetric measurements by means of hot wire anemometry and time-resolved particle image velocimetry. Three possible mechanisms for the so-called "separation-stall noise" have been identified in addition to a clear link between far-field noise, surface pressure, and velocity fields in the noise generation.

DOI: 10.1103/PhysRevFluids.4.123902

\section{INTRODUCTION}

There are many examples in which an airfoil operates close to stall, either intentionally to maximize lift, or inadvertently, such as in the case of a wind turbine blade experiencing a sudden gust. Airfoils operating close to stall generally suffer a degradation in aerodynamic performance. Moreover, at these conditions, airfoils are also well known to be particularly noisy, characterized by large increases in low-frequency noise [1]. This paper is a detailed experimental study into the characteristics and mechanisms of this additional increase in low-frequency noise.

Increasing the airfoil angle of attack produces an increase in the adverse pressure gradient over the airfoil suction side, which then induces a separation of the boundary layer from the surface. As long as this separation is incipient, the separated layer is still able to reattach to the surface in a relatively short distance. In some cases, reattachment can occur in less than $1 \%$ of the chord [2]. Increasing the incidence angle further causes the point of reattachment to move farther downstream. Transition may occur near this reattachment point, triggering the onset of a turbulent boundary layer that convects past the trailing edge and then scatters into sound. In general, if the flow conditions change from laminar to turbulent, the size of the separated wake can be reduced and the onset of separation can be delayed since the energizing effect of the outer layer on the turbulent boundary layer is greater than in the laminar case due to the turbulent mixing motion [3]. In a general situation, where the turbulence is generated within the airfoil boundary layer itself and then interacts with the trailing edge to generate noise, the noise generation mechanism is well understood and can be predicted with reasonable accuracy using classical flat plate theory once the boundary layer pressure statistics are known near the trailing edge [4]. Once the boundary layer becomes separated from the trailing edge there is evidence that the radiated noise can still be determined from the pressure statistics close to the trailing edge [5]. However, the cause of these pressure fluctuations is much less well understood. This paper provides further evidence that the noise radiated under near-stall conditions arises from trailing edge scattering. However, its main focus is to investigate the cause of these pressure fluctuations, which has received comparatively little attention in the literature.

\section{A. Previous work}

Airfoil separation noise is one example of a class of airfoil noise-generating mechanisms, categorized as airfoil self-noise, in which (predominantly) hydrodynamic pressure fluctuations convect over the trailing edge, identified by Brooks et al. in 1989 [1]. Separation noise is characterized by a significant increase (typically more than $10 \mathrm{~dB}$ [6]) in low-frequency noise [7] relative to the noise at low angles of attack, when the boundary layer is attached.

Airfoil noise at near-stall conditions has been measured in a number of studies [1,5,8-10]. These measurements have been used to develop models of the radiated noise. The best known of these has arisen from the empirical BPM (Brooks, Pope, and Marcolini) model [1], which provides predictions of the radiated noise at $90^{\circ}$ to the trailing of a NACA0012 airfoil over a range of angles of attack, including stall conditions. The main input parameters to this model are the displacement and boundary layer thicknesses at the trailing edge, which are also determined from empirical expressions. We note, however, that the notion of boundary layer thickness is less well defined for a separated shear layer. The implicit assumption, therefore, is that the noise spectrum is completely determined by eddy structures within the separated flow, whose size is determined by the boundary layer thickness. According to Brooks et al. [1] the contribution to the total noise due to the separated 
portion of the turbulent boundary layer on the suction side, based on the suction side boundary layer thickness, is predicted by the formula

$$
\mathrm{SPL}_{\alpha}=10 \log \left(\frac{\delta_{s}^{*} M^{5} L \bar{D}_{h}}{r_{e}^{2}}\right)+B\left(\frac{\mathrm{St}_{s}}{\mathrm{St}_{2}}\right)+K_{2},
$$

where $\delta_{s}^{*}$ is the displacement thickness on the suction side, $M$ is the Mach number, $L$ is the spanwise extent wetted by the flow (airfoil width), $\bar{D}_{h}$ is a directivity function, $r_{e}$ is the retarded observer distance, $B$ is the spectral shape function, which depends on the Strouhal number based on the displacement thickness on the suction side $\left(\mathrm{St}_{s}=\frac{f \delta_{s}^{*}}{U}\right)$ and on the peak Strouhal number $\mathrm{St}_{2}$ where the trailing edge noise is maximum, and $K_{2}$ is an amplitude function, depending on the angle of attack and Mach number. More details about the model can be found in Ref. [1].

Moreau et al. [8] have developed an analytical model for predicting airfoil separation noise based on experimental measurements of the wall pressure spectra and spanwise coherence lengths for NACA0012 and NACA65-(12)10 airfoils. More recently, Schuele and Rossignol [9] have proposed a model based on the TNO-Blake formulation in which the surface pressure fluctuations are determined from the steady and unsteady boundary layer velocity statistics via the solution to Poisson's equations for incompressible flow. The model was used to determine the radiated noise due to the separated boundary layer of a DU-96-W-180 airfoil. It was shown to achieve good levels of agreement with experimental data in the midfrequency range. Suryadi and Herr [10] have extended the study on the same airfoil in order to better predict the low-frequency range by means of a modification of the correlation lengths. Finally Bertagnolio et al. [5] have developed an empirical model for the surface pressure spectrum, based on a series of curve fittings of the surface pressure spectra data obtained from many different airfoil profiles and Reynolds numbers. This model requires only the separation point location as an input parameter and was shown to provide predictions that are in good agreement with various noise measurements. However, some discrepancies were observed in the low-frequency range for some particular configurations. The precise range of frequencies for which separation noise is most prominent depends on the physical extent of the separated area, which is a strong function of the chord length. For example, as reported by Moreau et al. [8], the noise associated with stall conditions for an airfoil of chord length $c=0.15 \mathrm{~m}$ is in the range of frequencies $f \approx 10^{2}-10^{3} \mathrm{~Hz}$. Further evidence of the importance of the chord length for separation noise can be found in Paterson et al. [7], who found significant levels of correlation between far-field and surface pressure microphones. They estimated the cross-correlation zero-crossing time delay among far-field and surface pressure microphones. For moderate angles of attack, this time delay monotonically decreases moving the surface pressure sensor location downstream along the chord approaching the trailing edge. This is an evidence of noise produced in correspondence to the trailing edge. In deep stall conditions, they have shown that, once you get closer to the trailing edge, this time delay starts reducing less, so that the related estimate of convective velocity of disturbances results in a very high value, higher than the freestream velocity. This is unlikely and suggests that the noise production process occurs also upstream of the trailing edge (allowing a different distance over which should be estimated the convective velocity, given the time delay), so that the dimension of the airfoil, and its chord, are now relevant for the noise generation. However, while the previously described models establish the link between the surface pressure near the trailing edge, due to hydrodynamic pressure fluctuations, and the far-field acoustic radiation, none of these studies have elucidated the mechanism of surface pressure fluctuations due to a separated boundary layer. This is the main objective of the current paper.

\section{B. Objectives and scope of the paper}

This paper is a detailed investigation into the relationship between velocity fluctuations in the boundary layer and shear layer, the surface pressure fluctuations near the trailing edge, and the far-field noise due to the NACA-65 family of airfoils over a wide range of angles of attack, including pre- and poststall conditions. Simultaneous far-field and surface pressure measurements were made 
in order to establish the link between them. Time-resolved particle image velocimetry (TRPIV) and hot wire anemometry (HWA) were also used in combination with the unsteady surface pressure measurements in order to understand the mechanisms of separation/stall noise on airfoils. The Reynolds number $(\mathrm{Re})$ for the current study is in the range $\mathrm{Re} \approx 2 \times 10^{5}-4 \times 10^{5}$.

This paper is organized as follows: In Sec. II the possible separation/stall noise generation mechanisms are reviewed. The airfoil geometry is described in Sec. III, and the experimental setup and measurement techniques are described in Sec. IV. In Sec. V far-field noise, unsteady surface pressure, and velocity field measurements are presented and their interrelationships discussed. Conclusions and a summary of the main findings are presented in Sec. VI.

\section{SEPARATION NOISE MECHANISMS}

A separated flow developing over an airfoil surface is a complex phenomenon, which may involve a transition to a fully turbulent flow and the occurrence of instabilities and shed vorticity. Two distinct flow regimes can be identified: the freestream, where the velocity is close to the undisturbed state, and a separating boundary layer region, involving flow recirculation. These two zones are separated by a shear layer, which is prone to being unstable and rolling up to form vortices [11]. The characteristics of these vortices can depend on the static surface pressure distribution over the airfoil, which generally depends on geometry, Reynolds number, and angle of attack [11,12]. Based on elementary considerations of the dynamics of separating shear layers, three possible distinct noise generation mechanisms can be identified:

(1) Coherent structures convected in the detached shear layer. When a separated flow is developing over an airfoil, a spanwise vortex roll-up process of the separated shear layer is triggered [13]. This generates vortex structures oriented in the spanwise direction that while convected downstream tend to break down and tilt towards the streamwise direction [14] The transit of these coherent structures in the shear layer passing over the trailing edge will induce an unsteady hydrodynamic pressure over the airfoil surface, which then scatters at the trailing edge and radiates to the far field [Fig. 1(a)]. In a recent paper by Berk et al. [15], particle image velocimetry (PIV) velocity data of the flow field were combined with simultaneous measurements of the surface pressure on a NACA0012 airfoil to demonstrate the causal link between instances of high (or low) pressure on the surface with coherent structures passing over the trailing edge. A similar procedure will be adopted here to demonstrate the existence of coherent structures in the shear layer and their link to the far-field radiation due to a NACA65 airfoil at high angle of attack.

(2) Instabilities in the detached shear layer. Wavelike instabilities, such as Kelvin-Helmholtz instabilities [16], can occur in the separated shear layer [Fig. 1(b)]. Such waves have been shown to be theoretically possible from unstable solutions of the Orr-Sommerfeld equation, whose eigenfrequencies have been shown to closely correspond with peaks in the hydrodynamic pressure spectrum on the airfoil surface [17]. These largely convected modes will induce surface pressure fluctuations on the surface and subsequently radiate as sound.

(3) Shear layer flapping. In conditions of incipient separation, it has been found $[18,19]$ that the shear layer can exhibit a flapping motion [Fig. 1(c)]. This is generally found to be a low-frequency phenomenon, usually occurring at a nondimensional frequency of $(\mathrm{St} \approx 0.02-0.03$ ), where the Strouhal number is defined as $\mathrm{St}=\frac{f c \sin (\alpha)}{U_{\infty}}$, based on the projected width obstructing the flow $[c \sin (\alpha)]$, where $c$ is the airfoil chord and $\alpha$ is the angle of attack. The flapping phenomenon is associated with a periodic switching between stalled and unstalled conditions downstream to the leading edge. Evidence for flapping as a low-frequency noise phenomenon is presented below in Sec. V I.

\section{AIRFOIL CONFIGURATIONS}

The experimental investigation of airfoil stall noise presented in this paper is based on the NACA65-(12)10 family of airfoils, which are high-performance airfoils, designed to maximize 
Coherent structures in shear layer

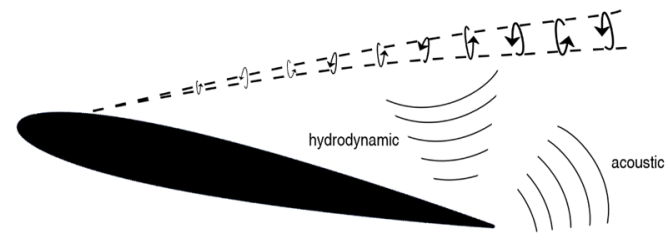

(a)
Shear layer instabilities

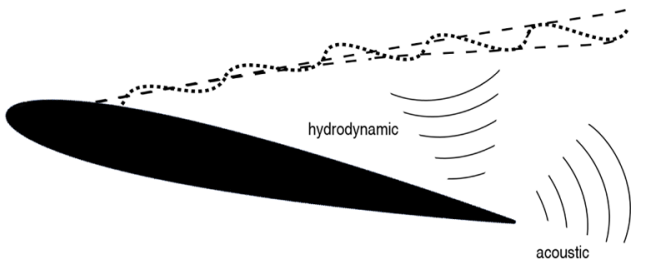

(b)

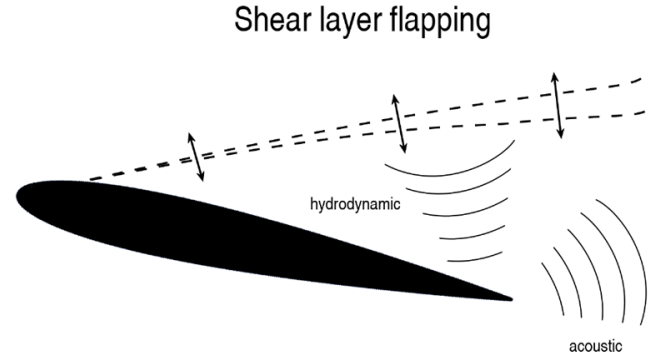

(c)

FIG. 1. Possible separation noise mechanisms associated with (a) coherent structures in the shear layer, (b) shear layer instabilities, and (c) shear layer flapping.

the region of laminar flow over their surface [20]. They are often used in cascades and turbines. The NACA65-(12)10 airfoil under investigation has a chord of $0.15 \mathrm{~m}$ and a span of $0.35 \mathrm{~m}$. To avoid possible tonal noise generation due to Tollmien-Schlichting instabilities, occurring when the airfoil is in a laminar condition, the flow near the leading edge was tripped by means of a band of sandpaper at a location of around one-third of the chord $(5 \mathrm{~cm})$ from the leading edge on both the pressure and the suction sides. The sandpaper covered the entire span of the airfoil and had a thickness $t=0.5 \mathrm{~mm}$ and a width in the streamwise direction $w=1 \mathrm{~cm}$.

\section{EXPERIMENTAL SETUP}

\section{A. Facility}

All noise and flow measurements were performed in the open jet wind tunnel facility at the Institute of Sound and Vibration Research at the University of Southampton. Photographs of the facility and the experimental setup are shown in Fig. 2. A detailed description of the wind tunnel can be found in Chong et al. [21]. It is housed inside the university's large anechoic chamber, with dimensions of $8 \mathrm{~m} \times 8 \mathrm{~m} \times 8 \mathrm{~m}$, whose walls are acoustically treated with glass wool wedges whose cutoff frequencies are $80 \mathrm{~Hz}$. The nozzle dimensions are $0.5 \mathrm{~m}$ in height and $0.35 \mathrm{~m}$ in width. This nozzle height together with the chord length of $0.15 \mathrm{~m}$ ensures that the downwash deflection of the jet is sufficiently small to readily allow measurements at poststall conditions. The ratio between the geometrical angle $\alpha_{\text {geom }}$ and the effective angle of attack $\alpha_{\text {eff }}$, i.e., after flow deflection has been taken into account, can be estimated from the relationship derived by Brooks et al. [22], which applied to the current configuration is

$$
\zeta=\frac{\alpha_{\mathrm{geom}}}{\alpha_{\mathrm{eff}}} \approx 1.5,
$$




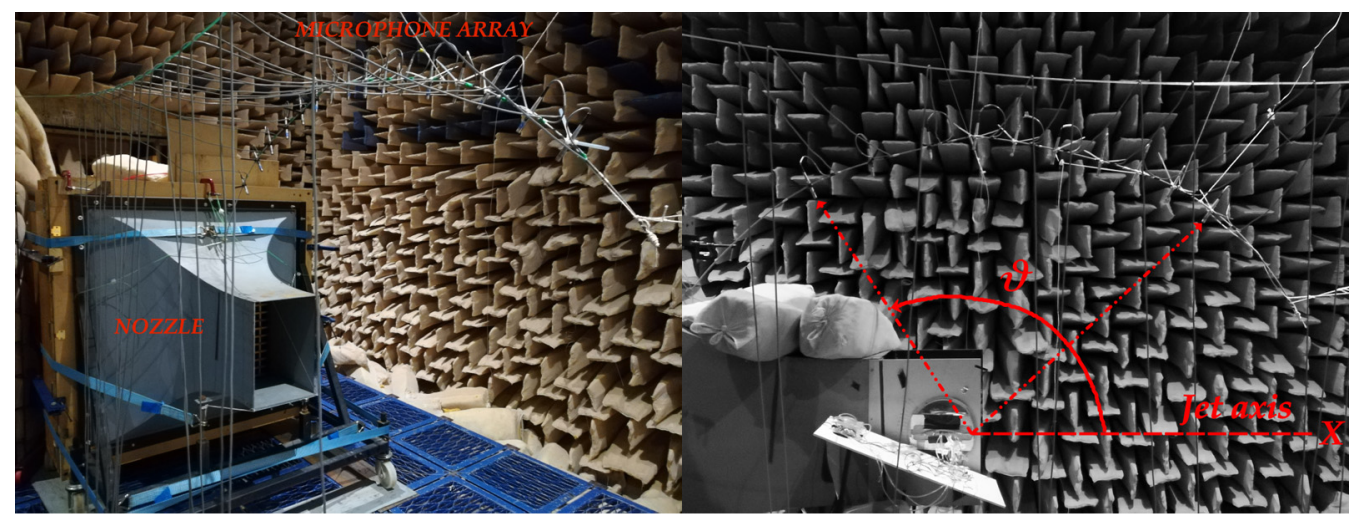

(a)

(b)

FIG. 2. (Left) Open wind tunnel and acoustic setup inside the ISVR's anechoic chamber. (Right) Microphone emission angles.

where $\zeta=(1+2 \sigma)^{2}+\sqrt{12 \sigma}, \sigma=\left(\frac{\pi^{2}}{48} \frac{c}{H}\right)^{2}, c$ the airfoil chord, and $H$ the nozzle height. The airfoil is held in place by two side plates, which also maintain the two-dimensionality of the flow, attached to the side walls of the nozzle. The leading edge of the airfoil was $0.15 \mathrm{~m}$ (one chord) downstream of the nozzle lip.

The velocity for all the measurements was set to a value of $20 \mathrm{~m} / \mathrm{s}$ or $40 \mathrm{~m} / \mathrm{s}$ corresponding to a value of Reynolds number $(\operatorname{Re}) \operatorname{Re} \approx 2 \times 10^{5}$ and $\operatorname{Re} \approx 4 \times 10^{5}$.

\section{B. Noise measurement}

Noise measurements were made using a polar array of 10 half-inch condenser microphones (B $\& \mathrm{~K}$ type 4189), located at a distance of $1.2 \mathrm{~m}$ from the trailing edge at the midspan plane of the airfoil, as shown in Fig. 2 (right). The microphones emission angles range from $40^{\circ}$ to $130^{\circ}$, relative to the airfoil trailing edge and downstream direction of the jet axis. Measurements were made at a sampling frequency of $40 \mathrm{kHz}$ for a duration of $20 \mathrm{~s}$. The total number of samples is then $8 \times 10^{-5}$, and the PSD estimate window length is 4096 samples. This combination of parameters gives a frequency resolution $\Delta f$ of $1 / 0.1024=9.8 \mathrm{~Hz}$. The normalized variance of the spectral estimate is equal to $\frac{1}{2 \Delta f T}$, where $\Delta f T$ is the so-called "BT product." For this combination of parameters the normalized variance of the spectral estimate is therefore 0.003 , which is negligible.

\section{Steady and unsteady pressure on airfoil surface}

Pressure measurements on the airfoil surface where made via a number of capillary tubes that run below the airfoil surface between pressure taps, along the airfoil chord, and along the span close to the trailing edge, and a number of T-junctions connected to miniature microphones. More specific information about the arrangement of pressure taps and tubes can be found in Sec. V B The microphones are $2.5 \mathrm{~mm}$ diameter omnidirectional electret condenser microphones (Knowles Electronics 206 FG-3329-P07). On the other side of the T junction are capillary tubes approximately $3 \mathrm{~m}$ in length to avoid reflections. Surface pressure measurements were also acquired simultaneously to the far-field noise for a duration of $20 \mathrm{~s}$ at a sampling frequency of $40 \mathrm{kHz}$. The total number of samples is then $8 \times 10^{-5}$, and, given the same PSD window length as for the noise measurements, similar observations about the variance of the spectral estimate can be made. Special care was given to the sealing of the microphone within the block, which was found to affect the pressure measurements. The miniature microphones were calibrated in situ against a reference B\&K $\frac{1}{4}$ inch 


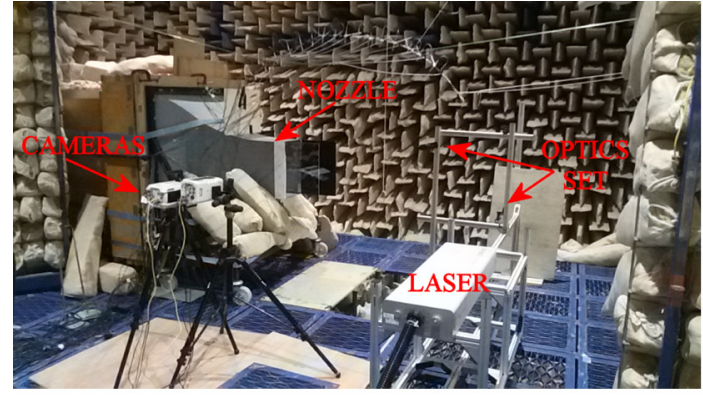

(a)

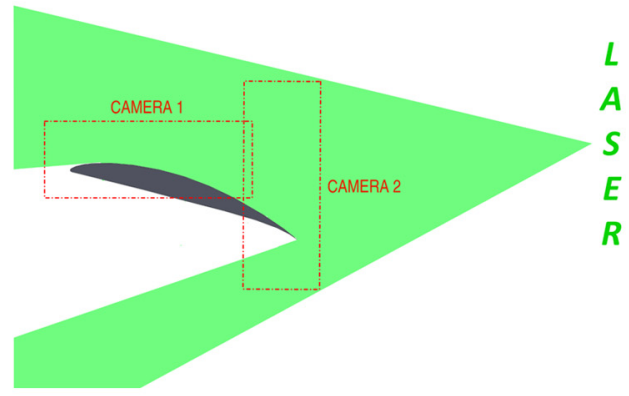

(b)

FIG. 3. (Left) TRPIV setup inside the ISVR's anechoic chamber. (Right) Sketch showing the PIV cameras' fields of view (dashed lines) and the total framed area.

condenser microphone by means of an in-duct loudspeaker. This arrangement of capillary tubes was also used to measure the steady pressure coefficient $C_{p}$, by replacing the microphones with membrane-based piezo-resistive amplified pressure sensors (First Sensor HCLA0050) having an operating pressure in the range $0 \pm 50$ mbar with a ratiometric analog output signal.

\section{PIV measurement}

The steady and unsteady velocity fields around the airfoil at angles of attack close to stall were investigated by means of time-resolved particle image velocimetry (TRPIV) at a frequency of $4 \mathrm{kHz}$ and based on the acquisition of roughly 20000 images, corresponding to $5 \mathrm{~s}$ of data, by means of the setup shown in the photograph in Fig. 3 (left). A Nd:YLF laser capable of a high-repetition rate of up to $10 \mathrm{kHz}$ was used to generate a light beam, which was then converted by a system of cylindrical and spherical lenses into a light sheet of $1 \mathrm{~mm}$ thickness with which to illuminate the vertical plane along the chord of the airfoil in the streamwise direction. Two high-speed Phantom v641 cameras, set at a resolution of $1024 \times 512$ pixels $(0.5 \mathrm{MP})$, were used to frame the area around the airfoil, mostly on the suction side, in a T-shape configuration; see Fig. 3 (right). The cameras have a $10 \mu \mathrm{m}$ sensor pixel size and are equipped with an $85 \mathrm{~mm}$ focal length lens. The flow was seeded using a Martin Magnum 1200 smoke machine located at the inlet of the centrifugal fan of the wind tunnel, which provided a uniform particle distribution of the flow from the nozzle with diameters of roughly $1 \mu \mathrm{m}$. The average particle image size was approximately 2.2 pixels, and the number of particles per pixel $\left(N_{p p p}\right)$ was about 0.038 , which is close to the optimal value prescribed by Willert and Gharib of $\left(N_{p p p}=0.035\right)$ [23] and in agreement with the recommendations of Raffel et al. [24] and Cierpka et al. [25] $\left(0.03<N_{p p p}<0.05\right)$. Finally, the magnification factor $M$ was about $6.7 \mathrm{pixel} / \mathrm{mm}$ (roughly $0.15 \mathrm{~mm} / \mathrm{pixel}$ ). The calibration between the object domain and the image domain was performed using the recommended procedure described in Adrian and Westerweel [26]. The PIV images were processed using digital cross-correlation analysis [23]. A multigrid/multipass algorithm [27], with an iterative image deformation [28-31], was used to compute the instantaneous velocity fields having a final interrogation window size of $32 \times 32$ pixels with an overlap factor of $75 \%$. In order to eliminate spurious vectors, a vector validation algorithm, based on a regional median filter [32], with a kernel region of $3 \times 3$ vectors, and group removing, was applied.

\section{RESULTS}

\section{A. Validation of measurement procedure}

By way of validation of the measurement procedure and of the wind tunnel, initial measurements were made of the noise due to a NACA0012 airfoil of $0.2 \mathrm{~m}$ chord at high angle of attack and compared against the spectra predicted by the BPM model [1], which provides estimates for the 


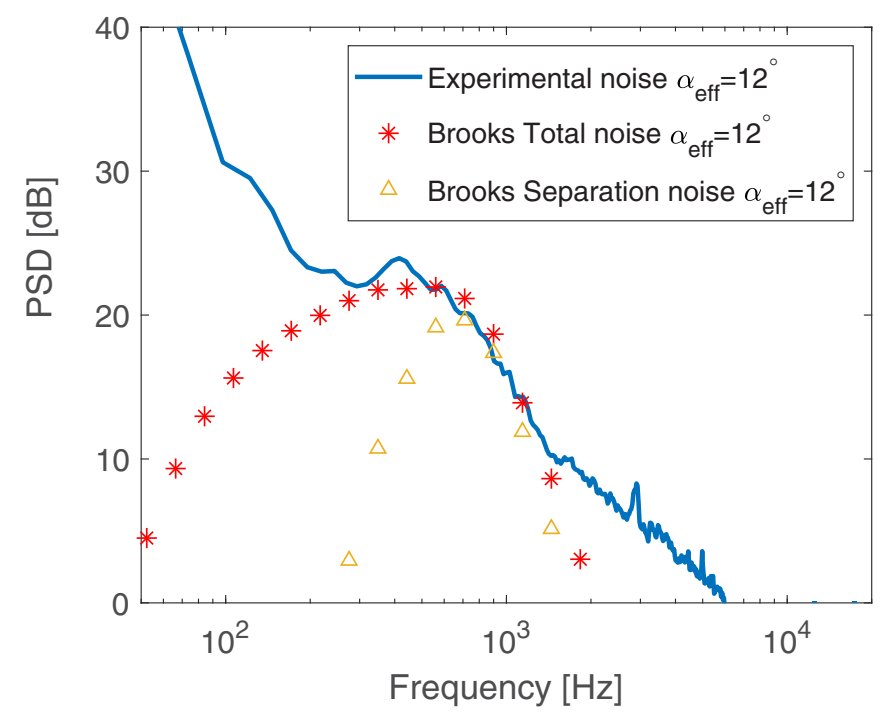

FIG. 4. Measured NACA0012 noise spectra vs Brooks et al. [1] predicted total self-noise and separation noise contribution at $\alpha_{\text {eff }}=12^{\circ}$.

$1 / 3$-octave band frequency spectrum for the different "self-noise" mechanisms, including separation noise, associated with a NACA0012 airfoil. Figure 4 shows the comparison between the measured and predicted sound pressure level spectra at a location $90^{\circ}$ from the trailing edge, a freestream velocity equal to $U_{\infty}=20 \mathrm{~m} / \mathrm{s}$, and an effective angle of attack of $\alpha_{\text {eff }}=12^{\circ}$, for which separation noise was found to be the dominant noise mechanism in the midfrequency range. The experimental noise spectral density is integrated over a bin width of $4.9 \mathrm{~Hz}$. In this figure both the predicted overall noise and separation noise alone are plotted. The spectral hump due to separation predicted by Brooks et al. can be clearly observed in the measured spectrum in the frequency range between about 250 and $1500 \mathrm{~Hz}$. Below this frequency the measured spectra are dominated by facility noise. The reason for the discrepancy above $1500 \mathrm{~Hz}$ is not clear. Nevertheless, the level of agreement in the region of the spectral hump is sufficient to validate the measurement procedure and facility. We now investigate the characteristics of separation noise due to a different airfoil, the NACA65-(12)10, at a flow speed of $40 \mathrm{~m} / \mathrm{s}$.

\section{B. Steady pressure on airfoil surface}

Before investigating the characteristics of the radiation from the NACA65-(12)10 airfoil at a high angle of attack, we first assess the steady pressure coefficient $C_{p}$ distribution along the midchord in order to determine the development of the separated flow areas and hence determine the angle of attack at which stall conditions are established. The $C_{p}$ distribution was estimated from just five pressure taps along each of the pressure and suction sides of the airfoil chord, evenly distributed between $15 \mathrm{~mm}$ from the leading edge and $5 \mathrm{~mm}$ from the trailing edge. By way of validation of the measurement technique and to assess the accuracy of the pressure sensors, we have compared the measured $C_{p}$ with the one obtained from XFoil [33] but only for the range of angles at which XFoil gives a converged solution $\alpha \leqslant 10^{\circ}$. The comparison is shown in Fig. 5, and the agreement is good both in terms of shape and identification of flow separated regions ( $\operatorname{see} \alpha=10^{\circ}$ ) as the region of nearly constant static pressure [34]. Figure 5(b) shows now the measured $C_{p}$ only and, since the separation mainly occurs on the airfoil suction side, only $C_{p}$ on this side is shown in this figure for some representative angles of attack. 


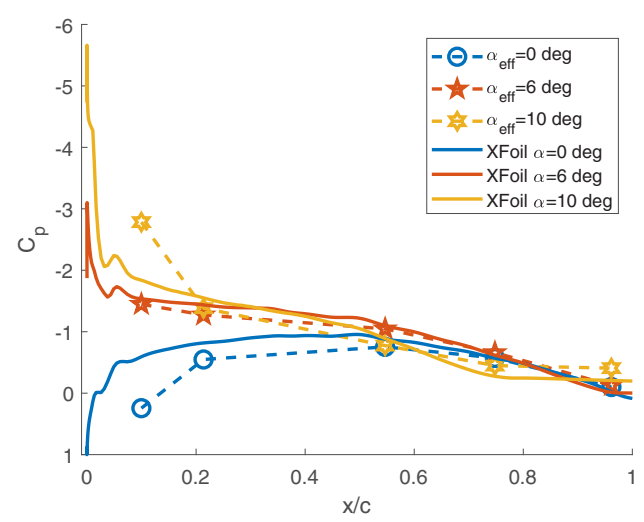

(a)

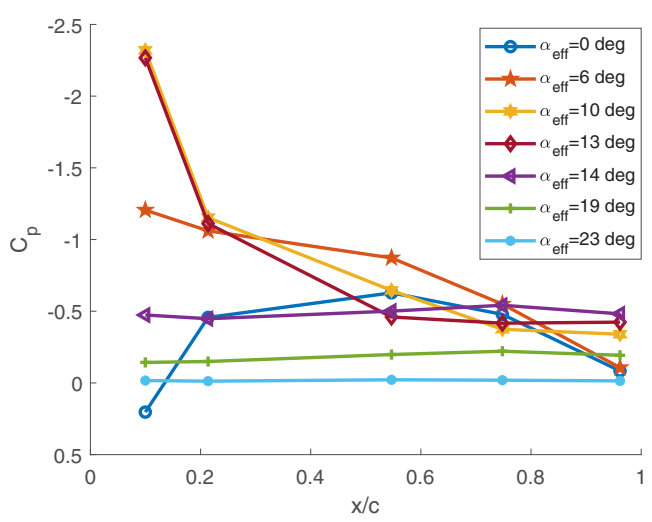

(b)

FIG. 5. NACA65(12)10 airfoil suction side. (Left) Comparison between the pressure distribution measured by pressure sensors and estimated by XFoil. (Right) Measured pressure distribution at different angles of attack. $U_{\infty}=40$.

At moderate angles of attack $\left(\alpha_{\text {eff }}=0^{\circ}-6^{\circ}\right)$, the flow can be seen to remain attached over the entire airfoil surface. At the angle of attack of $\alpha_{\text {eff }}=10^{\circ}$, a separated flow area is established close to the TE of the airfoil (the final $20 \%-25 \%$ of the chord). At $\alpha_{\text {eff }}=13^{\circ}$, at least $50 \%$ of the chord can be seen to be affected, while further increasing the angle of attack to $\alpha_{\text {eff }}=14^{\circ}$ causes the flow to become separated over the entire suction side surface, eventually becoming fully stalled at angles greater $\left(\alpha_{\mathrm{eff}}=19^{\circ}-23^{\circ}\right)$. A progressive and gradual upstream movement of the point of separation from the trailing edge towards the leading edge as $\alpha_{\text {eff }}$ is increased is usually classified as trailing-edge stall; see Ref. [35]. A trailing-edge stall generates a "soft" stall marked by a gradual "bending over" of the lift curve at maximum lift, as compared to the sharp, steep drop in $C_{L}$ for a leading-edge stall [35].

\section{Characterization of noise spectra}

Having made an initial assessment of the different flow conditions around the NACA65-(12)10 airfoil, we now consider how the radiated noise varies for these different flow regimes. The sound power level spectra PWL(f) obtained by summing the pressure spectra across the microphones and assuming cylindrical radiation, according to the procedure discussed in Ref. [36], was determined for different effective angles of attack in the range $\left[0^{\circ}: 26^{\circ}\right]$. The noise spectral densities are integrated over a bin width of $4.9 \mathrm{~Hz}$. For the sake of clarity, however, only the angles $0^{\circ}, 6^{\circ}$, $10^{\circ}, 13^{\circ}, 14^{\circ}, 19^{\circ}$, and $23^{\circ}$ are shown in Fig. 6 . For the range of angles of attack corresponding to fully attached flow over the suction side surface, $\left(\alpha_{\text {eff }}=0^{\circ}-6^{\circ}\right)$ (as shown from the pressure distribution in Fig. 5), relatively small increases in noise can be observed with increasing angles of attack. At $\alpha_{\text {eff }}=10^{\circ}$, where separation at the trailing edge begins to occur, a sudden increase in noise is observed with a peak frequency of about $f=500 \mathrm{~Hz}$. Further increasing the angle of attack to $\alpha_{\text {eff }}=13^{\circ}$ and $\alpha_{\text {eff }}=14^{\circ}$ causes an additional and significant increase in noise over a lower frequency range. At $\alpha_{\text {eff }}=19^{\circ}$ and above, the airfoil is fully stalled, and the maximum noise level decreases but its frequency bandwidth increases.

For angles of attack higher than $\alpha_{\text {eff }}=6^{\circ}$, the center frequency of the spectral hump can be observed to reduce as angle of attack increases, while the flow separated region increases its extension moving from the trailing edge towards the leading edge. This is consistent with the model due to Brooks et al. [1], who predicts the peak frequency based on Strouhal number defined with respect to the boundary layer thickness, or rather the extension of the flow separated region. These peak frequencies are highlighted by markers in Fig. 6. 


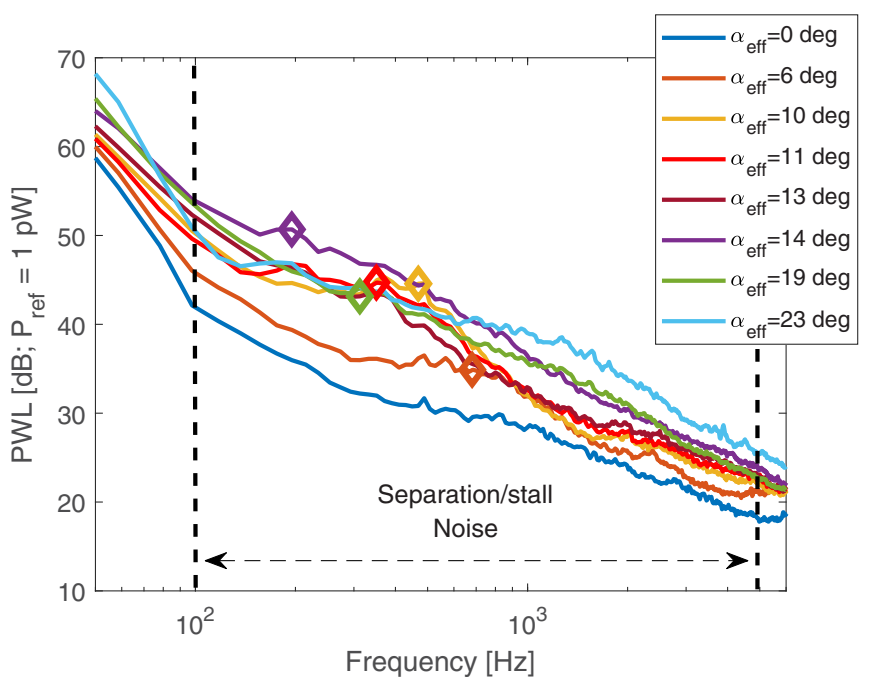

FIG. 6. Noise results for the NACA65(12)10 airfoil at $U_{\infty}=40 \mathrm{~m} / \mathrm{s}$. Sound power level at different angles of attack. The locations of the center frequencies of the spectral humps are shown as the angle of attack changes from $\alpha_{\text {eff }}=0^{\circ}$ to $\alpha_{\text {eff }}=14^{\circ}$. The reference power is $P_{\text {ref }}=1 \mathrm{pW}$.

A clearer representation of the behavior of the radiated noise with angle of attack can be seen in Fig. 7, which shows the variation in sound pressure level versus frequency and angle of attack relative to the noise at $0^{\circ}$. Four different regimes can be clearly identified:

(I) Low angle of attack, $\alpha_{\text {eff }}<8^{\circ}$. The flow is still attached at the trailing edge, such that increasing angle of attack causes the boundary layer to become thicker and more energetic and the noise spectrum to shift towards lower frequencies. In this range of angles the relative noise spectrum is nearly flat.

(II) Midangles of attack, $8^{\circ}<\alpha_{\text {eff }}<14^{\circ}$. The flow separates from the trailing edge, and the noise spectrum is concentrated over a relatively narrow frequency bandwidth, which is between

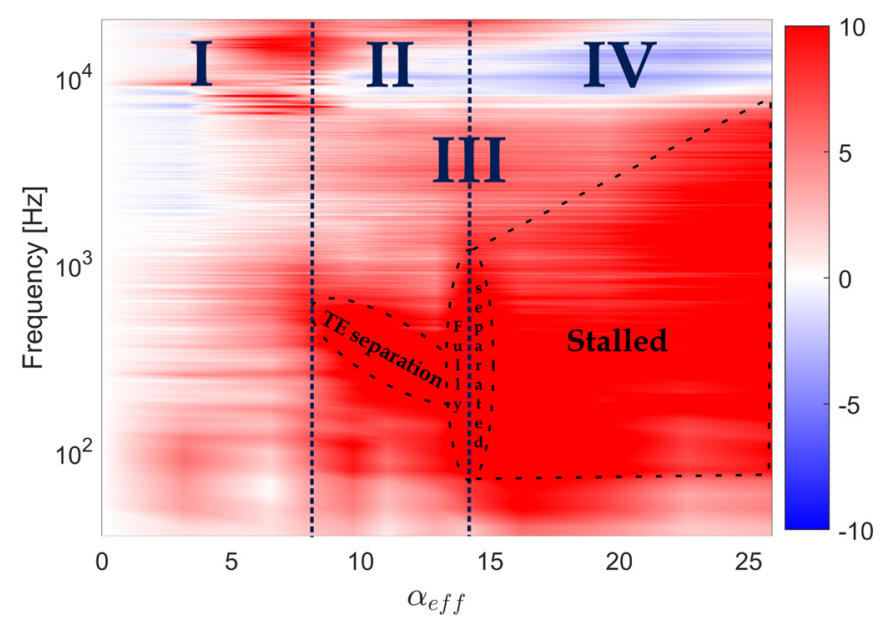

FIG. 7. Noise increase with the angle of attack compared to $\alpha_{\text {eff }}=0^{\circ}$ for the NACA65(12) 10 airfoil at $U_{\infty}=40 \mathrm{~m} / \mathrm{s}$. Four different zones are highlighted: turbulent boundary layer (TBL) noise (low angle of attack) (I), flow separated noise (II), critical angle $\alpha_{\text {eff }}=14^{\circ}$ (III), flow stalled noise (IV). 


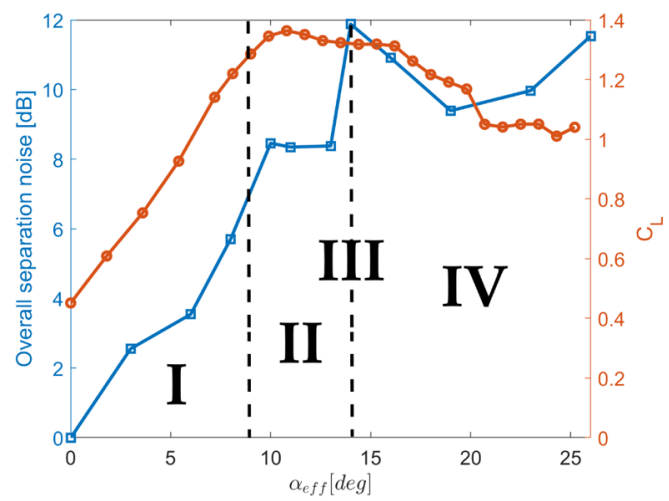

(a)

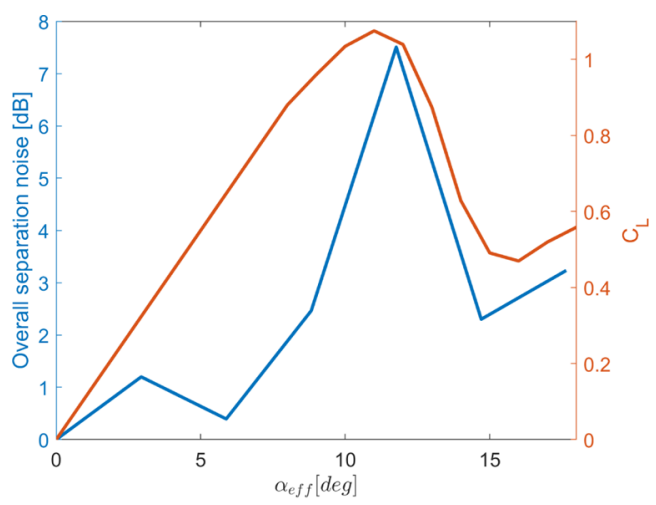

(b)

FIG. 8. Noise results at $U_{\infty}=40 \mathrm{~m} / \mathrm{s}$. Trend of the separation noise contribution to the overall noise at different angles of attack vs lift curve from pressure distribution: (a) NACA65, (b) NACA0012.

about 100 and $800 \mathrm{~Hz}$ in this case. In this range of angles the noise appears to be relatively insensitive to angle of attack.

(III) Critical angle of attack, $\alpha_{\mathrm{eff}}=14^{\circ}$. At this critical angle the point of separation has just reached the leading edge. At this condition the shear layer turbulence above the trailing edge is highest while the height of the shear layer above the trailing edge is lowest. This combination of conditions leads to maximum noise noise generation over the entire frequency range associated with the separation/stall noise while there is a relative noise reduction at high frequencies.

(IV) High angles of attack, $\alpha_{\text {eff }}>14^{\circ}$. The flow is stalled on the entire airfoil suction side. While the shear layer turbulence increases with increasing angle of attack, the distance of the shear layer from the trailing edge also increases. Since it is well known that the hydrodynamic pressures generated in the shear layer fall off exponentially with distance (e.g., see the TNO-Blake model [37-39]), the noise generation begins to fall with increasing angle of attack. This decrease is limited only by the flow approaching a condition similar to a bluff body as the angles of attack become extremely high.

Based on the previous different regimes, a distinction can be made between the following:

Separation noise: Characterized by an unsteady flow close to the surface and for which the noise increase is restricted to a relatively narrow frequency bandwidth with a spectral hump whose center frequency can be associated with the thickness of the separated layer.

Stall noise: Characterized by an unsteady flow which is farther from the surface and radiates hydrodynamic waves that interact with the body and for which the noise increase broadens to a wide range of frequencies.

\section{Relationship between separation noise and lift}

The previous section has demonstrated a close relationship between airfoil noise at any arbitrary angle of attack and the "state" of the boundary layer. It is therefore reasonable to assume that the variation in overall noise and total lift on the airfoil with angle of attack follow similar trends. Figure 8 (left) shows a comparison between the increase in overall noise from $\alpha_{\text {eff }}=0^{\circ}$ (left-hand scale) and the lift coefficient (right-hand scale) versus effective angle of attack. The overall noise was calculated over a bandwidth of between $10^{2}$ and $5 \times 10^{3} \mathrm{~Hz}$ and the lift measured on the same airfoil model by means of three-component force transducer (Kyowa, LSM-B-SA1, Rated Capacity: $10 \mathrm{~N}$ ) in the open-type wind tunnel at the University of Nottingham with a sampling rate of $0.5 \mathrm{kHz}$. The frequency bandwidth for the noise was chosen to correspond to the full range of frequency associated with the separation noise (more restricted to low frequency) and stall 
noise (more broadband). Also shown in this figure are the angles of attack identified in Sec. VC delineating the four regions of behavior.

Similar variations in lift and overall noise versus angle of attack can be observed. In region I both noise and lift increase with increasing angle of attack. Separation from the trailing edge occurring in region II limits the increase in lift with increasing angle of attack, and the overall noise remains roughly constant. At the critical angle in region III, a sharp peak in the noise can be observed corresponding to the angle at which the flow just becomes separated at the leading edge and overall lift begins to reduce. Finally in region IV both noise and lift begin to reduce with increasing angle of attack as the flow becomes increasing detached from the trailing edge. At extremely high angles of attack $\alpha_{\text {eff }}>20^{\circ}$ the lift and the emitted noise start increasing again. This can be associated with the airfoil being approached by the flow basically as a bluff body.

Figure 8 provides strong evidence for the close association between lift and overall noise for the NACA65 airfoil over a wide range of angle attack. It also identifies the condition of maximum noise. To assess the generality of these findings an identical analysis was performed on the noise and lift data for a NACA0012 airfoil and the comparison shown in Fig. 8 (right). The lift data were taken from the report by Sheldahl and Klimas [40], choosing the dataset associated with the Reynolds number $\left(\operatorname{Re}=7 \times 10^{5}\right)$ closest to our current one.

With the scales chosen appropriately the variation in overall noise and lift with angle of attack are in close agreement. In the case of the NACA0012 airfoil the stall behavior is more complex since this airfoil geometry exhibits a trailing edge stall at all Reynolds numbers but a combined leading edge/trailing edge stall at intermediate Reynolds numbers (possibly between $\operatorname{Re} \approx 3 \times 10^{4}$ and $\operatorname{Re} \approx 3 \times 10^{6}[41]$ ), when the airfoil starts to stall with a turbulent boundary layer moving upstream from the trailing edge but the flow breakdown is completed by an existing laminar separation zone in the leading edge failing to reattach [41]. This is characterized by a "semirounded" lift-curve peak followed by a relatively rapid decrease in lift [42]. Accordingly, the variation in noise and lift around the peak is now much sharper than in the case of the NACA65 airfoil, which has a much gentler poststall behavior.

\section{Simultaneous far-field and surface pressure measurements}

It is now well established that when the boundary layer is attached at the trailing edge, the far-field pressure spectra $S_{p p}$ and hydrodynamic surface pressure spectrum $S_{q q}$ close to the trailing edge are proportional to within a factor of the coherence length $l_{y}(\omega)$ [4]. In this case, therefore, the trailing edge is the origin of the far-field radiation. However, once the flow becomes separated at the trailing edge, it is no longer clear that the far-field noise originates from the trailing edge and that this relationship is still valid.

In this section we present simultaneous measurements of the surface pressure and far-field noise spectrum over a range of angles of attack. The frequency range over which their spectral shapes are similar therefore corresponds to the range at which the trailing edge is the source of noise. We first present the coherence between them to identify the frequency range over which the far-field radiation at a single point is causally related to the surface pressure at a single point on the trailing edge.

Figure 9 (left) shows the coherence between the pressure measured at the pressure tap on the airfoil suction side closest to the trailing edge $(5 \mathrm{~mm}$ upstream) and a point in the far field. At the two low angles of attack of $0^{\circ}$ and $6^{\circ}$, the coherence between the surface pressure and far-field pressure is negligible over the entire frequency range. The reason for this poor coherence is because the eddy size, which scales on the boundary layer thickness, is much smaller than the airfoil span. The far-field noise is therefore due to a large number of these small-scale eddies interacting with the trailing edge. At $10^{\circ}$ a small hump in the coherence spectrum at about $500 \mathrm{~Hz}$ can be observed with a maximum coherence of about 0.2 . At $13^{\circ}$ the "hump" now peaks at a value of 0.4 and occurs at the low frequency of about $250 \mathrm{~Hz}$. At much higher angles of attack of $14^{\circ}, 16^{\circ}$, and $19^{\circ}$, the peak frequency is now shifted to the much lower frequencies of about $50 \mathrm{~Hz}$. Since in these conditions 


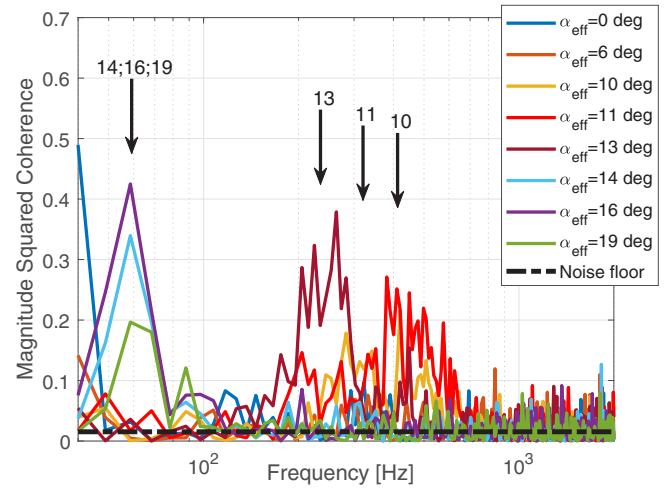

(a)

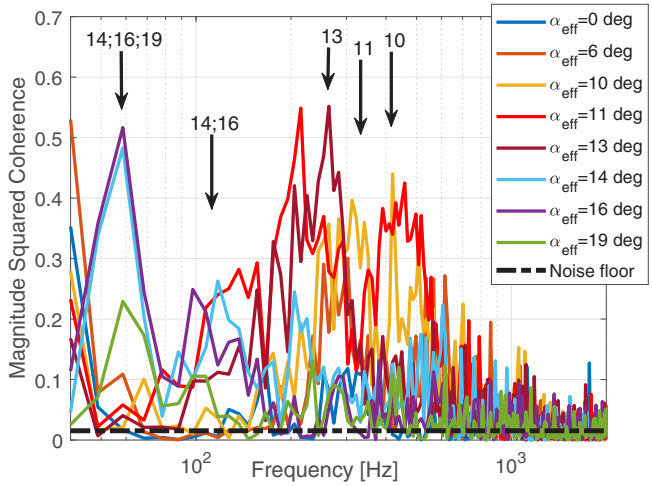

(b)

FIG. 9. Coherence between far-field noise and surface pressure at TE for the NACA65(12)10 airfoil at different angles of attack and $U_{\infty}=40 \mathrm{~m} / \mathrm{s}$ on (a) suction side and (b) pressure side. The dashed lines in panels (a) and (b) represent the statistical noise floor. The windowing was 4096 data points.

the noise increase is over a much broader frequency range, as shown in Fig. 7, it can be speculated that the trailing edge is no more the only source of noise for a fully separated/stalled airfoil. This conclusion was also reached in the classic paper by Paterson et al. [7], who, by measuring the time delay between the far-field noise and different microphones along the chord, showed that the noise at high angle of attack originates somewhere upstream of the trailing edge.

\section{E. Estimation of size of "flow structures" from coherence value}

At any flow condition, the far-field noise at a single frequency is due to a number of similar flow structures of approximate average spanwise length $l_{y}(\omega)$ passing over the trailing edge, where $l_{y}(\omega)$ is the frequency-dependent spanwise coherence length $l_{y}(\omega)=\int_{-\infty}^{\infty} \gamma_{p_{s} p_{s}}^{2}(\omega, \Delta r) d(\Delta r)$, where $\gamma_{p_{s} p_{s}}^{2}(\omega, \Delta r)$ is the spanwise coherence between the surface pressures separated by a spanwise distance $\Delta r$ close to the trailing edge. For an airfoil of span $L$ there are therefore $L / l_{y}(\omega)$ uncorrelated sources along the span (taken to the nearest integer), each of which radiates to the far field upon interaction with the trailing edge. Consider now the coherence $\gamma_{p_{s} p_{f}}^{2}(\omega)$ between the surface pressure $p_{s}$ at a point close to the trailing edge and the acoustic pressure $p_{f}$ at a single far-field location, defined by

$$
\gamma_{p_{s} p_{f}}^{2}(\omega)=\frac{\left|S_{p_{s} p_{f}}(\omega)\right|^{2}}{S_{p_{s} p_{s}}(\omega) S_{p_{f} p_{f}}(\omega)},
$$

where $S_{p_{s} p_{f}}(\omega)$ is the cross-spectral density between the surface pressure $p_{s}(\omega)$ and far-field pressure $p_{f}(\omega)$ and where $S_{p_{s} p_{s}}(\omega)$ and $S_{p_{f} p_{f}}(\omega)$ are the autospectral densities of the surface pressure and far-field pressures, respectively. The far-field pressure $p_{f}(\omega)$ is the sum of $L / l_{y}(\omega)$ statistically identical uncorrelated sources whose strength is proportional to the surface pressure $p_{s}(\omega)$ close to the trailing edge, each of which radiates to a far-field observer with a near-identical transfer function $G(\omega)$. At a single frequency, therefore, the radiated pressure $p_{f}(\omega)$ may be related to the surface pressure $p_{s}(\omega)$ by

$$
p_{f}(\omega)=\frac{L}{l_{y}(\omega)} G(\omega) p_{s}(\omega)
$$

However, since the pressures are random, we consider the spectral density of the radiated pressure, $S_{p_{f} p_{f}}(\omega)=E\left\{p_{f}(\omega) p_{f}^{*}(\omega)\right\}$. Substituting Eq. (2) into this expression and assuming that 
each of the $L / l_{y}(\omega)$ sources $p_{s}(\omega)$ are mutually uncorrelated, gives

$$
\begin{aligned}
S_{p_{f} p_{f}} & =E\left\{p_{f}(\omega) p_{f}^{*}(\omega)\right\}=\sum_{n=1}^{L / l_{y}(\omega)} \sum_{n^{\prime}=1}^{L / l_{y}(\omega)} E\left\{p_{s}\left(\omega, r_{n}\right) p_{s}^{*}\left(\omega, r_{n^{\prime}}\right)\right\} G\left(\omega, r_{n}\right) G^{*}\left(\omega, r_{n^{\prime}}\right) \\
& =\frac{L}{l_{y}(\omega)}|G(\omega)|^{2} S_{p_{s} p_{s}}(\omega),
\end{aligned}
$$

where $|G(\omega)|$ represents the value of $G\left(\omega, r_{n}\right)$ averaged over $r_{n}$ and

$S_{p_{s} p_{s}}(\omega)=E\left\{p_{s}\left(\omega, r_{n}\right) p_{s}^{*}\left(\omega, r_{n}\right)\right\}$ is the surface pressure autospectral density. This result simply means that the overall mean-square radiated pressure is the sum of $L / l_{y}(\omega)$ identical uncorrelated radiated pressures on the surface and is entirely consistent with classical result due to Amiet except that his expression includes the factor $L l_{y}(\omega)$ as a result of double integration along the span and the use of a different radiation function.

Similarly, substituting Eq. (2) into the expression for the cross-power spectral density $S_{p_{s} p_{f}}(\omega)=$ $E\left\{p_{s}(\omega) p_{f}^{*}(\omega)\right\}$ gives

$$
S_{p_{s} p_{f}}(\omega)=\overline{G(\omega)} S_{p_{s} p_{s}}(\omega)
$$

Substituting Eqs. (3) and (4) in Eq. (1) for the coherence yields

$$
\gamma_{p_{s} p_{f}}^{2}(\omega)=\left[\frac{l_{y}(\omega)}{L}\right]^{2} .
$$

Clearly, therefore, the coherence between the surface pressure and far-field pressure is unity when the surface pressure is fully coherent across the span. Equation (5) provides a simple and obvious interpretation of the coherence function as being equivalent to the number of uncorrelated sources. It also provides a means of estimating the coherence length from the near field/far-field coherence function,

$$
l_{y}(\omega)=L \sqrt{\gamma_{p_{s} p_{f}}^{2}(\omega)}
$$

The "humps" in the coherence spectrum of relatively high coherence in these figures are therefore indicative of strong "two-dimensionality" of the flow structure as might be expected from laminar separated flow.

\section{F. Simultaneous far-field noise and surface pressure measurement on pressure side}

Figure 9(b) (right) shows the coherence spectra between a single far-field receiver position and a point on the surface near the trailing edge $(5 \mathrm{~mm}$ upstream) but now measured on the pressure side where there is no flow separation. The same peak frequencies as in Fig. 9(a) can be observed but with generally higher coherence levels. This observation provides evidence for scattering at the trailing edge of the separated flow, which affects both the suction and pressure sides. The reason for the generally higher coherence between the far-field microphone and the pressure-side pressure, compared to the suction side, is because the pressure side is not contaminated by nonradiating hydrodynamic pressure fluctuations, which mostly occur on the suction side.

Confirmation of the higher levels of hydrodynamic pressure on the suction side is shown in Figs. 10(a) and 10(b), which show a direct comparison between the autospectral densities of the surface pressures on the pressure side and the suction side at the two angles of attack of $\alpha_{\text {eff }}=10^{\circ}$ and $\alpha_{\text {eff }}=14^{\circ}$. These two angles of attack correspond to the onset of separation at the trailing edge and leading edge, respectively. The autospectral densities of the unsteady surface pressure are integrated over a bin width of $4.9 \mathrm{~Hz}$. At $\alpha_{\text {eff }}=10^{\circ}$ the suction-side surface pressure exceeds the pressure side by nearly $15 \mathrm{~dB}$ in the frequency range where separation noise is dominant. At the higher angle of attack, $\alpha_{\text {eff }}=14^{\circ}$, where the flow is completely separated, the pressures on both sides of the airfoil are roughly similar in the low-frequency bandwidth where separation noise is 


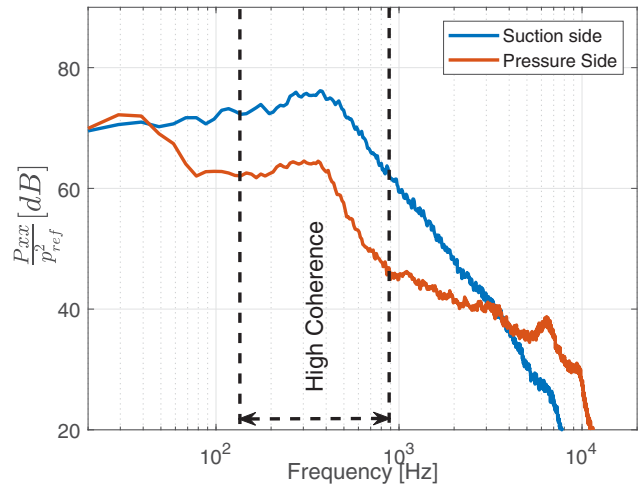

(a)

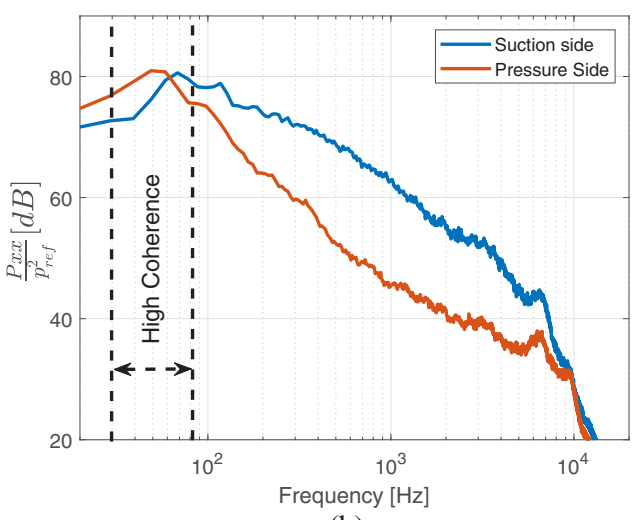

(b)

FIG. 10. Autospectral density of the unsteady surface pressure field at the TE on both sides of the airfoil at (a) $\alpha_{\text {eff }}=10^{\circ}$ and (b) $\alpha_{\text {eff }}=14^{\circ}$.

dominant. This may be due to very long wavelengths $(>1 \mathrm{~m})$ associated with the noise due to flow separation in this case, which will diffract around the trailing edge causing both sides of the airfoil to be similar.

\section{G. Comparison of surface and far-field pressure spectra}

Further demonstration of the causal link between the unsteady surface pressure close to the airfoil trailing edge and the far-field noise for separated flows can be obtained by simply comparing their increase in spectra levels for various angles of attack above the spectrum at $\alpha_{\text {eff }}=0^{\circ}$. The autospectral densities of far-field noise and unsteady surface pressure are both integrated over a bin width of $4.9 \mathrm{~Hz}$.

These comparisons between the surface pressure at the pressure tap closest to the trailing edge ( $5 \mathrm{~mm}$ upstream) and the microphone $90^{\circ}$ to the trailing edge are shown in Fig. 11 for the four representative angles of attack $\alpha_{\mathrm{eff}}=3^{\circ}, 10^{\circ}, 16^{\circ}$, and $23^{\circ}$. At $\left(\alpha_{\mathrm{eff}}=3^{\circ}\right)$ where the flow is attached, the increase in $\triangle \mathrm{PSD}$ above the spectra at $0^{\circ}$ is generally less than $5 \mathrm{~dB}$ in both surface and farfield spectra. At $\alpha_{\text {eff }}=10^{\circ}$ as the flow separates at the airfoil trailing edge the two plots exhibit strong similarity in the frequency range associated with separation noise $\left(f \approx 10^{2}-10^{3}\right)$, clearly establishing the link between them. At $\alpha_{\text {eff }}=16^{\circ}$ even though the flow is fully separated over the airfoil surface the spectral shapes are similar within and beyond the frequency range where the coherence is high, again suggesting a causal link between them. However, at the much higher angle of $\left(\alpha_{\text {eff }}=23^{\circ}\right)$ spectral shapes match only over a very narrow low-frequency range with very poor agreement occurring at higher frequencies. This suggests that additional sources are likely to be present, other than at the trailing edge, at this high angle of attack.

By way of summary, therefore, the peak in the noise radiation spectrum characteristic of separation noise has two contributions:

(1) A spectral peak in the surface pressure spectrum, whose origin will be investigated in Sec. V J.

(2) An increase in the coherence length in the same narrow frequency band, leading to an increase in radiation efficiency of the hydrodynamic pressure fluctuations near the trailing edge.

\section{H. Velocity fields}

In this section we attempt to identify the sources of separation noise through measurements of the unsteady flow around the airfoil. TRPIV was used to quantify the unsteady velocity field at a freestream velocity $20 \mathrm{~m} / \mathrm{s}$ at an effective angle of attack of $\alpha_{\mathrm{eff}} \approx 11^{\circ}$ corresponding to the 


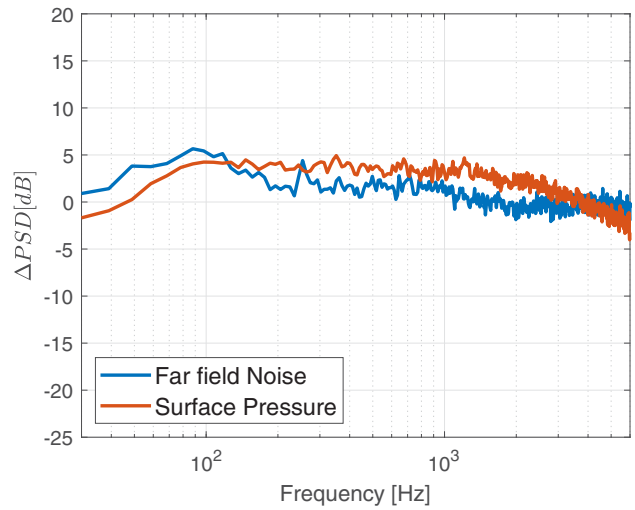

(a)

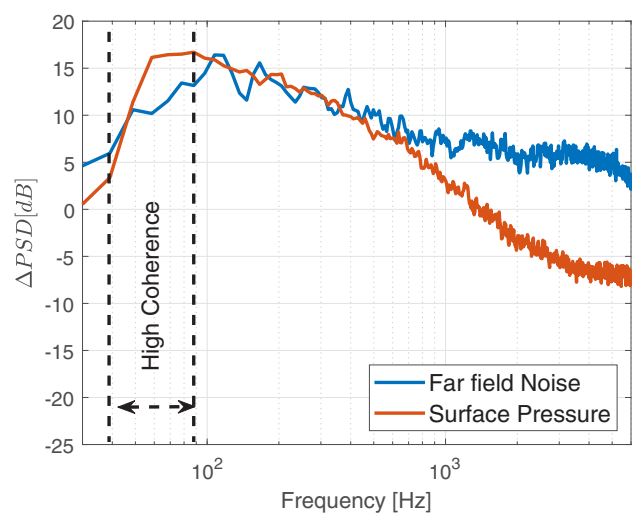

(c)

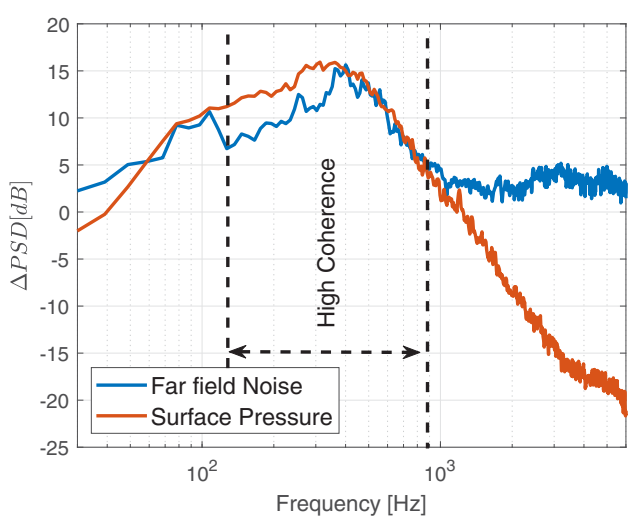

(b)

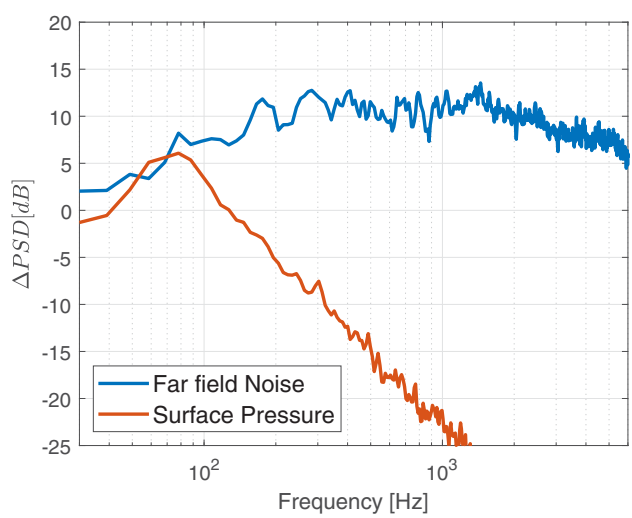

(d)

FIG. 11. Increase of the unsteady surface pressure at TE and far-field noise autospectral densities above the spectrum at $\alpha_{\text {eff }}=0^{\circ}$ at (a) $\alpha_{\text {eff }}=3^{\circ}$, (b) $\alpha_{\text {eff }}=10^{\circ}$, (c) $\alpha_{\text {eff }}=16^{\circ}$, and (d) $\alpha_{\text {eff }}=23^{\circ}$. $U_{\infty}=40 \mathrm{~m} / \mathrm{s}$.

condition at which trailing edge separation is well established. The maximum velocity was limited to $20 \mathrm{~m} / \mathrm{s}$ due to constraints in the PIV setup. The Reynolds number is therefore not invariant to the noise measurements, but we assume that the flow field is not strongly affected by this dissimilarity even in terms of transition to a turbulent behavior since the critical Reynolds number is well established to be above $\operatorname{Re} \approx 5 \times 10^{5}$ [43]. Figures 12(a) and 12(b) show the contours of mean axial and transverse velocity components, while Figs. 12(c) and 12(d) show their corresponding root-mean-square (rms) values. The Reynolds shear stress and spanwise vorticity are plotted in Figs. 12(e) and 12(f). The extent of flow separation is evident in the plots of mean flow, where separation can be seen to occur at about $60 \%$ of the chord from the trailing edge. High levels of fluctuating velocity and vorticity identify the shear layer and the main sources of unsteadiness that can provide the source of noise.

\section{Causal relationship between boundary layer velocity fluctuations and unsteady surface pressure measurements}

Previous sections have established the trailing edge as the origin of the radiated noise due to separated flow. We now attempt to identify the sources of turbulence in the separated flow that are 


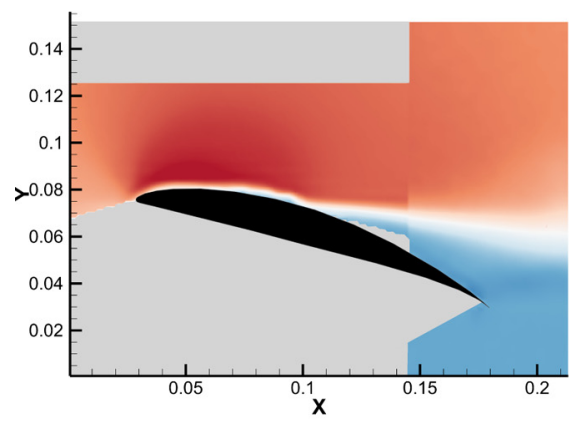

(a)

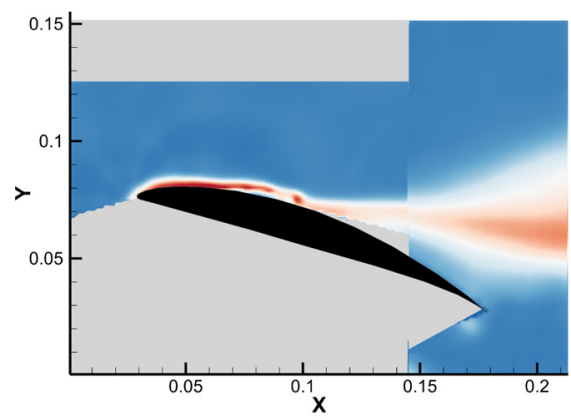

(c)

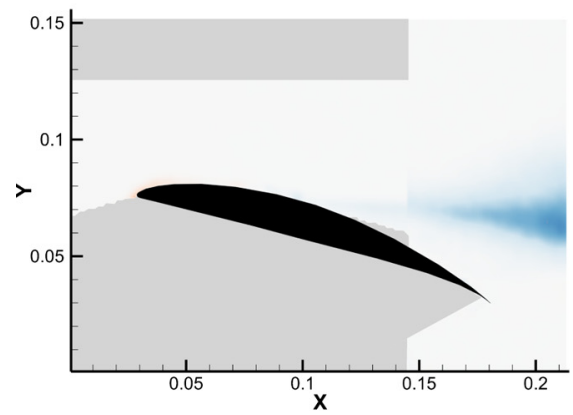

(e)

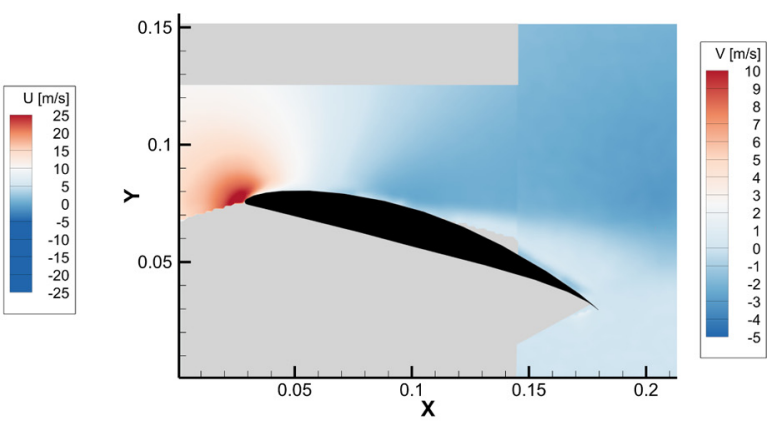

(b)

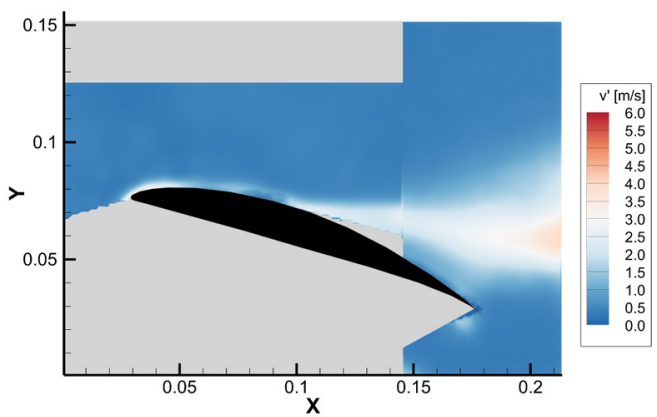

(d)

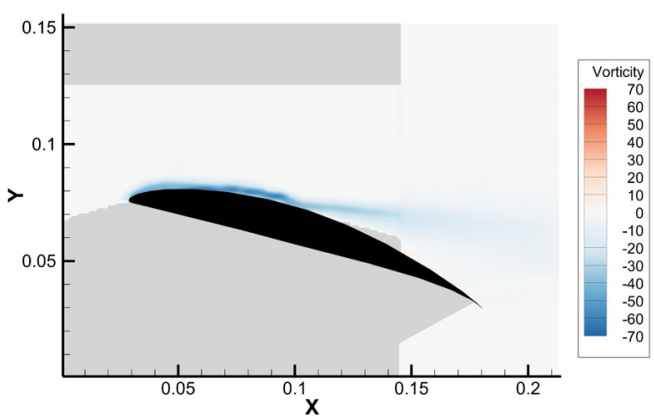

(f)

FIG. 12. Statistical description of the velocity field around the airfoil. (a) Mean streamwise velocity, $U$, (b) mean vertical velocity, $V$, (c) streamwise velocity fluctuations, $u^{\prime}$, (d) streamwise velocity fluctuations, $v^{\prime}$, (e) Reynolds stress, $\left(u^{\prime} v^{\prime}\right)$, and (f) mean spanwise vorticity, $\omega_{z}=v_{x}-u_{y}$ normalized by $U_{\infty} / c$. The units follow the SI metric system.

responsible for the unsteady pressure close to the trailing edge in the narrow frequency bandwidth in which separation noise dominates. Here we exploit the finding that separation noise occurs in a relatively small frequency bandwidth and must therefore be generated by reasonably coherent flow structures in the shear layer passing over the trailing edge. We therefore attempt to identify the sources of turbulent flow based on the assumption that they have maximum coherence with the surface pressure fluctuations near the trailing edge.

Surface pressure measurements $5 \mathrm{~mm}$ from the trailing edge were therefore made simultaneously with hot wire measurements of the streamwise velocity component at the airfoil trailing edge, as shown in the schematic diagram and photograph of the measurement in Figs. 13(a) and 13(b), respectively. Hot wire measurements were made at 40 to 50 vertical positions in increments of 


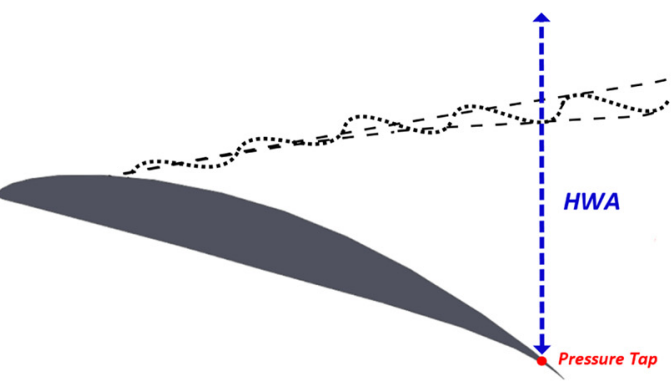

(a)

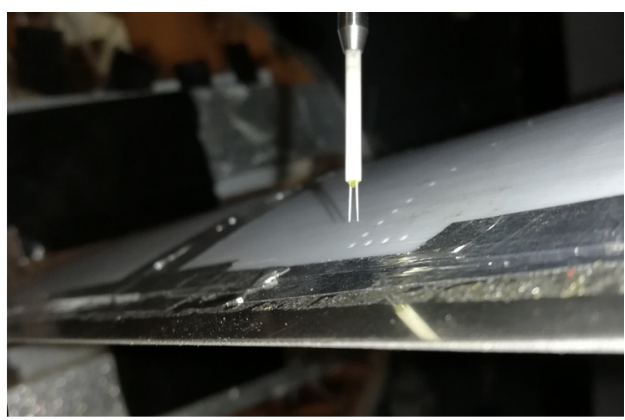

(b)

FIG. 13. Sketch and photo of the implementation of the simultaneous measurement of velocity [by means of hot wire anemometry (HWA)] and surface pressure.

$1.25 \mathrm{~mm}$ varying from $2 \mathrm{~mm}$ from the airfoil surface to the freestream region. The procedure was repeated for the three effective angles of attack of $\alpha_{\text {eff }} \approx 11^{\circ}, 13^{\circ}$, and $14^{\circ}$, which encompasses regions II and III identified in Sec. V C, ranging between developing separation to fully separated flow, at $U_{\infty}=20 \mathrm{~m} / \mathrm{s}$.

In Fig. 14, the variation in rms streamwise velocity with vertical height above the airfoil is shown at different angles of attack. The height of maximum rms velocity identifies the elevation of the shear layer, which divides the separated flow area from the freestream. As the angle of attack increases the height of the shear layer can be observed to move away from the airfoil surface. Small oscillations in the velocity profiles can be observed with a period of about $5 \mathrm{~mm}$. These features were found consistently on data obtained on different dates and airfoils. So far the reasons for these oscillations have not been clear, and further work is needed to establish their cause.

Figures 15(a)-15(c) show color contour maps of the coherence between the unsteady hot wire velocity data and the surface pressure, versus frequency and height above the surface at the trailing

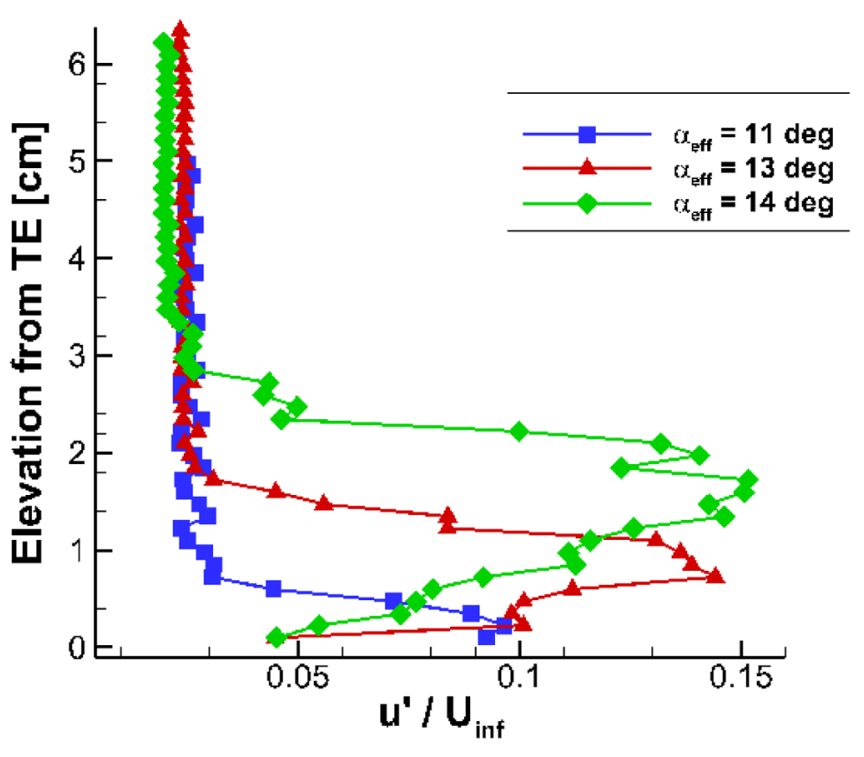

FIG. 14. Vertical profile of the standard deviation of the streamwise velocity component $u^{\prime}$ over the airfoil trailing edge at different angles of attack and at $U_{\infty}=20 \mathrm{~m} / \mathrm{s}$. 


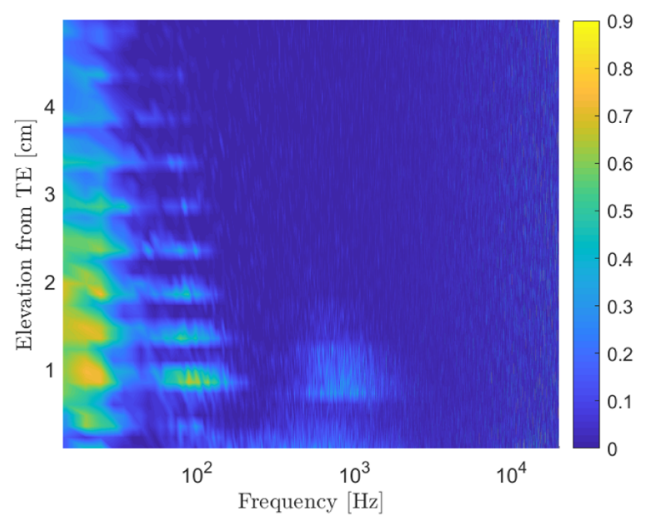

(a)

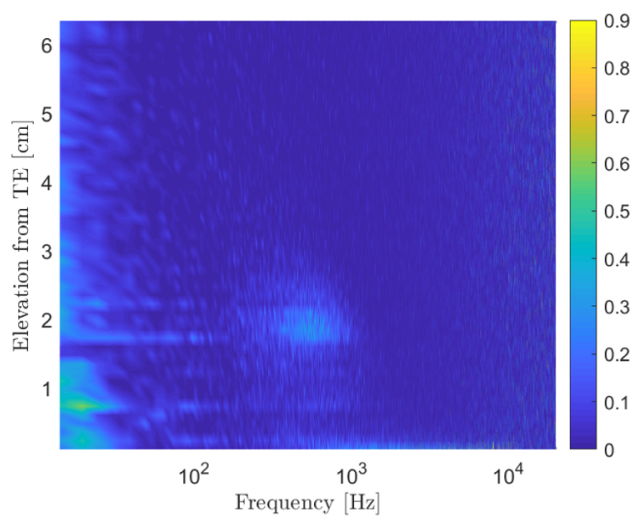

(b)

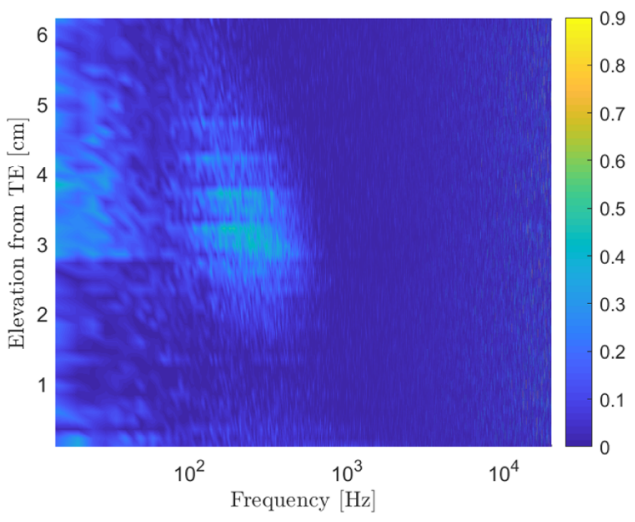

(c)

FIG. 15. Magnitude-squared coherence between velocity and surface pressure at the trailing edge as a function of frequency and spatial location (vertical distance from the airfoil surface) at $U_{\infty}=20 \mathrm{~m} / \mathrm{s}$. (a) $\alpha_{\text {eff }} \approx$ $11^{\circ}$, (b) $\alpha_{\mathrm{eff}} \approx 13^{\circ}$, (c) $\alpha_{\mathrm{eff}} \approx 14^{\circ}$.

edge. The results are shown at the three angles of attack of $11^{\circ}, 13^{\circ}$, and $14^{\circ}$, respectively. Again we associate the regions in the shear layer of high coherence (above 0.1 ) as being the sources responsible for the surface pressure fluctuations near the trailing edge and hence the far-field noise.

In Fig. 15(a) corresponding to $\alpha_{\text {eff }} \approx 11^{\circ}$ at least three distinct frequency regions of high coherence, and hence sources, can be identified. The region of most intense coherence with values above 0.5 occurs in the frequency range 10 to $20 \mathrm{~Hz}$ and is present at all positions above the shear layer. By virtue of the low frequency and spatial extent of this region we speculate that this is due to flapping of the shear layer. The Strouhal number $\mathrm{St}=\frac{f c \sin (\alpha)}{U_{\infty}}$ of this region based on the projected width obstructing the flow is close to $\mathrm{St}=0.03$, which has previously associated with flapping shear layers for incipient separation conditions [18,19], assuming a "turbulent" boundary layer (and a value $\mathrm{St}=0.02$ for a laminar boundary). The flapping has been linked to a periodic switching between stalled and unstalled states on the upper airfoil surface near the leading edge [19]. This low-frequency flapping motion generates hydrodynamic pressure fluctuations that are present throughout the flow and on the airfoil surface.

The second most intense region of high coherence in Fig. 15(a) occurs at frequencies close to $150 \mathrm{~Hz}$, which is also distributed through the shear layer. The third much weaker source occurs at frequencies around $1000 \mathrm{~Hz}$ and is concentrated just above the shear layer. In Sec. V J 1 below, PIV 
data for this angle of attack will be analyzed in detail to extract the dominant flow structures at these frequencies.

Increasing the angle of attack to $\alpha_{\text {eff }} \approx 13^{\circ}$ and $\alpha_{\text {eff }} \approx 14^{\circ}$ causes the source at $150 \mathrm{~Hz}$ to become weaker, while the high-frequency source shifts towards lower frequencies and moves farther away from the surface.

\section{J. Identification of the phenomena associated with separation noise}

The previous section has shown that separation noise may be associated with a number of distinctive regions within the shear layer of different characteristic frequencies. In this section dynamic mode decomposition is applied to the instantaneous velocity fields in order to identify the dominant flow "structures" and associated frequencies in the PIV data at $\alpha_{\text {eff }} \approx 11^{\circ}$ that most closely correspond to the dominant frequencies observed in Fig. 15.

\section{Dynamic mode decomposition}

Dynamic mode decomposition (DMD) is a common tool to identify and extract physically important features from experimental or numerical flow field data. This technique was first described in Ref. [44] and explained more fully by Schmid [45]. The technique decomposes time-resolved flow field data into Koopman modes [46], each of which is associated with a single characteristic frequency and growth or decay rate. It makes the use of the instantaneous flow field measurements at $K$ equispaced $(\Delta t)$ instances in time (referred to as the temporal dimension). These snapshots are arranged into vectors $\boldsymbol{v}\left(t_{k}\right) \equiv \boldsymbol{v}_{\boldsymbol{k}}$, whose spatial dimension $J$ corresponds to the number of grid points at which the velocity components are measured [for two-dimensional data $J=2\left(n_{x} \times n_{y}\right)$ ] and then used to construct a snapshot matrix:

$$
V_{1}^{K}=\left[v_{1}, v_{2}, \ldots, v_{k}, v_{k+1}, \ldots, v_{K-1}, v_{K}\right] .
$$

The output of the DMD is a Fourier-like expansion of the velocity field in terms of spatial modes in the form

$$
v_{k} \approx v_{k}^{\mathrm{DMD}} \equiv \sum_{m=1}^{M} a_{m} u_{m} e^{\left(\delta_{m}+i \omega_{m}\right)(k-1) \Delta t}
$$

for $k=1, \ldots, K$, where $u_{m}$ is the $m$ th DMD mode among the total of $M$ modes, $M$ being referred to as the spectral complexity, and $a_{m}, \delta_{m}$ and $\omega_{m}$ are the associated amplitudes, growth rates, and frequencies, respectively. The dimension $N$ of the vector subspace, generated by the M DMD modes and containing the approximation (7), is the spatial complexity which is $N \leqslant \min (J, M)$.

The standard DMD algorithm is based on the Koopman assumption that a linear mapping $\mathbf{A}$ connects each snapshot $v_{k}$ with the subsequent $v_{k+1}$ :

$$
v_{k+1}=\boldsymbol{A} v_{k}
$$

for $k=1, \ldots, K-1$.

The DMD complex frequencies $\delta_{m}+i \omega_{m}$ and mode shape functions $u_{m}$ are defined as the eigenvalues and eigenvectors of A. Since it is common that the number of snapshots is smaller than the number of grid points of each snapshot $(K \ll J)$, it is not efficient to compute $\mathbf{A}$ explicitly, and therefore a truncated singular value decomposition (SVD) is usually applied to the snapshot matrix to reduce the order of the snapshot matrix and eliminate noise. The SVD is of the form

$$
V^{k} \simeq W \Sigma T^{T}
$$

here $W^{T} W=T^{T} T=I$, where $I$ is the unit matrix, $W$ and $T$ are the left and right eigenvectors, and $\Sigma$ is the diagonal matrix containing the singular values $\sigma_{1}, \ldots, \sigma_{K}$. 


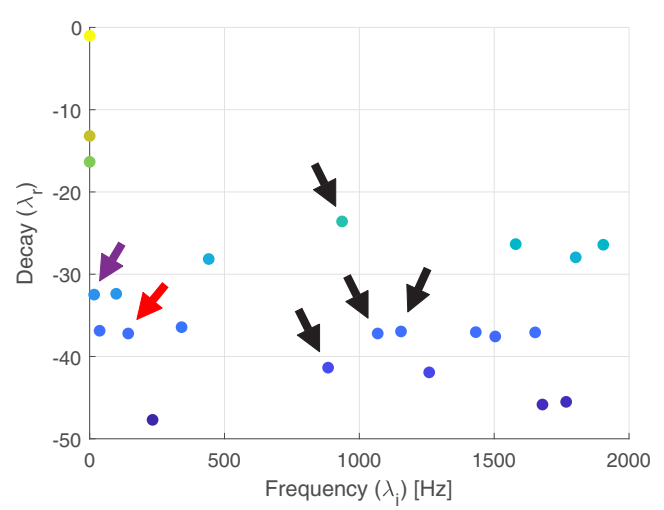

(a)

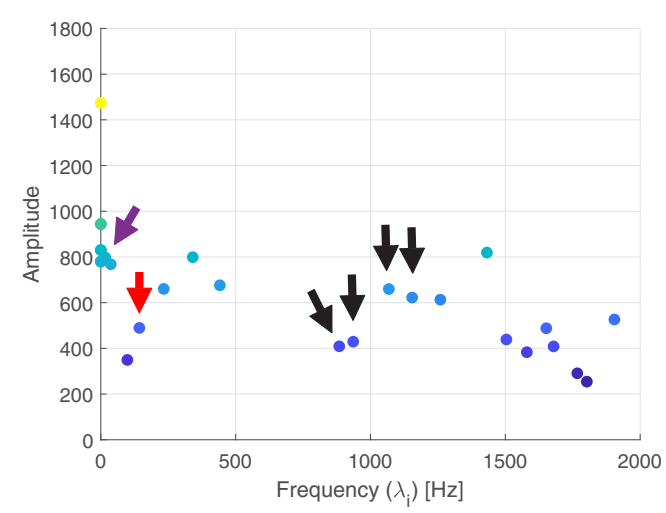

(b)

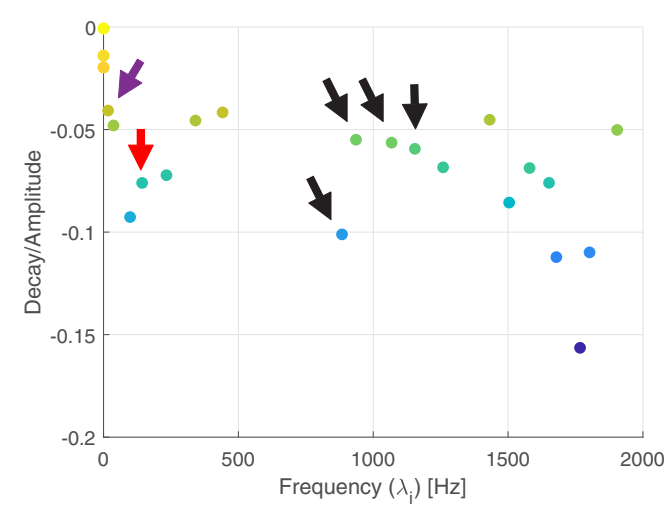

(c)

FIG. 16. DMD spectrum (a), amplitude absolute values (b), and ratio decay/amplitude (c) of the HODMD modes. $U_{\infty}=20 \mathrm{~m} / \mathrm{s}$; TRPIV sampling frequency $f_{s}=4 \mathrm{kHz}$. The color scale of the data points is a function of the relevance of the mode, where a lighter color is more relevant than a darker. The arrows highlight the modes that are examined below in more detail.

The reduced linear mapping matrix $\tilde{A}$ is defined as

$$
\tilde{A}=W_{r}^{T} A W_{r},
$$

where $W_{r}, \Sigma_{r}$, and $T_{r}$ represent truncated approximations to $W, \Sigma$, and $T$ with small singular values removed. The reduced mapping matrix $\tilde{A}$ can therefore be obtained from the relation $V^{k+1}=\tilde{A} V^{k}$, substituting Eq. (9), and by postmultiplication by $T_{r} \Sigma_{r}^{-1} W_{r}^{T}$, which gives

$$
\tilde{A}=V^{K+1} T_{r} \Sigma_{r}^{-1} W_{r}^{T} \text {. }
$$

The mode shape functions and corresponding complex DMD eigenvalues are finally obtained from nonzero eigenvalues $\mu_{m}$ and eigenvectors $\tilde{q_{m}}$ of $\tilde{A}$ using the expression

$$
\tilde{A} \tilde{q_{m}}=\mu_{m} \tilde{q_{m}} \text {. }
$$

Finally, the growth rates $\delta_{m}$ and frequencies $\omega_{m}$ are associated with the eigenvalues $\mu_{m}$ through

$$
\delta_{m}+i \omega_{m}=\log \left(\mu_{m}\right) / \Delta t .
$$




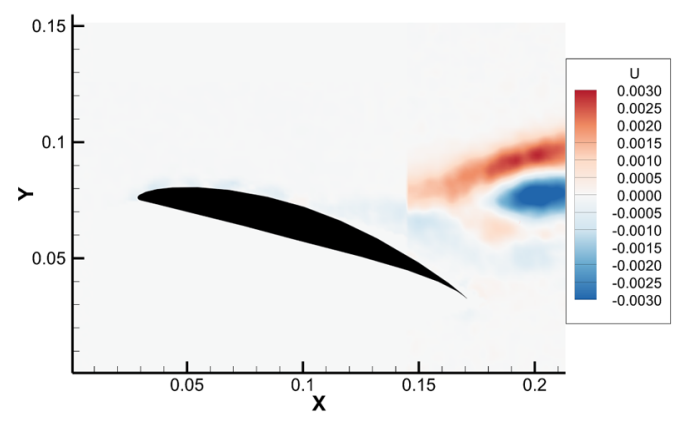

(a)

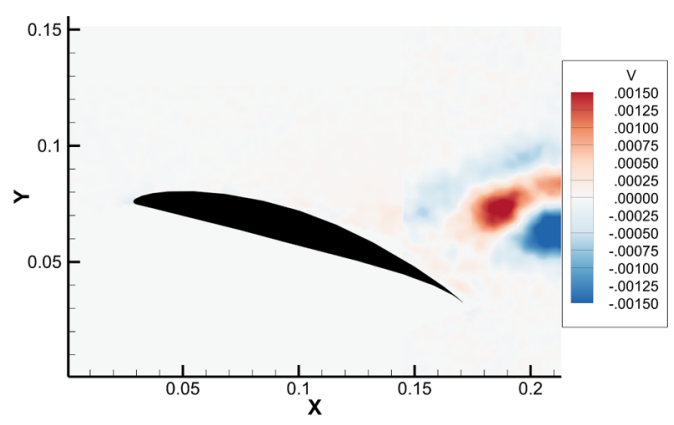

(b)

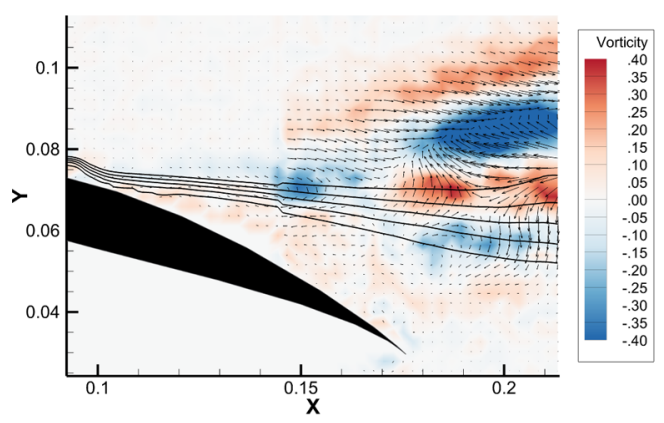

(c)

FIG. 17. High-order DMD mode associated with the frequency $f=16 \mathrm{~Hz}$. (a) Streamwise $U$ velocity component, (b) vertical $V$ velocity component, and (c) zoomed vorticity contour and velocity vectors with overlapped contour levels of the shear layer.

In this paper we use a recent extension of DMD, the higher-order DMD (HODMD) [47,48], which is favorable in highly noisy complex flows. This method is able to independently clean the data from noise in both spatial and temporal directions and relies on two dimension-reduction steps, based on truncated SVD with a set tolerance on the SVD error, prior to the computation of the DMD modes and eigenvalues. The underlying assumption is a more general higher order Koopman assumption that relates each snapshot with $d$ previous snapshots via some linear operators $A_{k}$ as

$$
v_{k+d} \simeq A_{1} v_{k}+A_{2} v_{k+1}+\cdots+A_{d} v_{k+d-1}
$$

for $k=1, \ldots, K-d$. Further details about the algorithm can be found in Ref. [48].

Figures 16(a)-16(c) show the decay rate (real part), amplitude, and ratio of decay rate to amplitude (DA ratio) versus modal frequencies (imaginary part), respectively. These were obtained from the HODMD, applied to 1000 instantaneous snapshots. The last figure shows a newly defined quantity that is useful in identifying the most significant modes associated with simultaneously low decay rate and high amplitude. Note that a small value of this ratio is an indicator of a possibly significant mode in the reconstruction of the time-varying flow field.

Figure 16 reveals about 20 discrete modes over the frequencies between 0 and $2000 \mathrm{~Hz}$ (Nyquist frequency). No clear dominant modes can be observed, which is consistent with the generally broadband character of the noise spectrum since no distinct dominant frequencies can be observed. We have therefore decided to investigate from the DMD spectrum the modes having an associated frequency close to the frequencies of high coherence between surface pressure, far-field pressure, and streamwise velocity, trying to identify any distinctive feature associated with these frequencies. The selected modes are highlighted by an arrow in Fig. 16 corresponding to the frequencies 


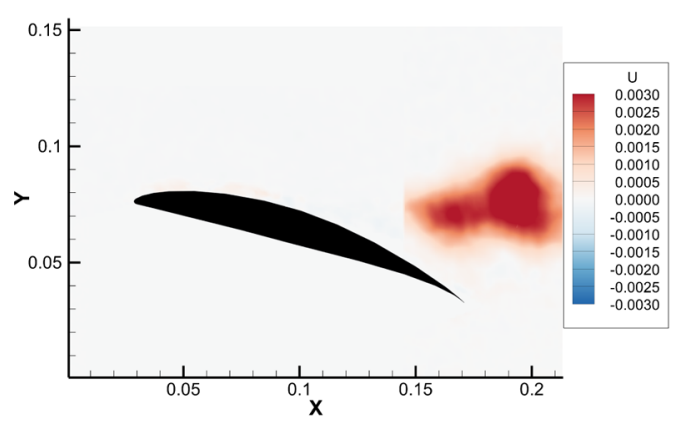

(a)

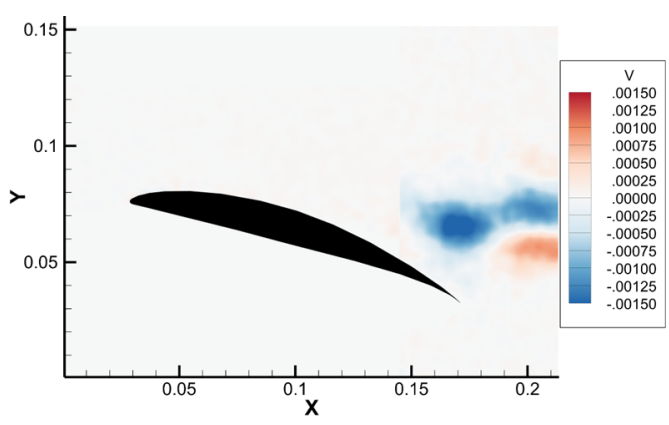

(b)

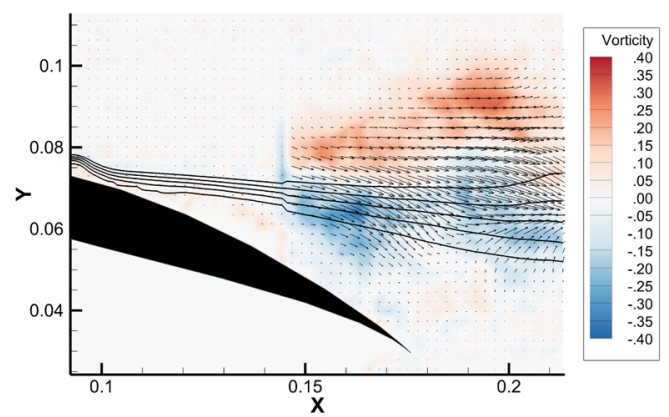

(c)

FIG. 18. High-order DMD mode associated with the frequency $f=143 \mathrm{~Hz}$. (a) Streamwise $U$ velocity component, (b) vertical $V$ velocity component, and (c) zoomed vorticity contour and velocity vectors with overlapped contour levels of the shear layer.

$f=16 \mathrm{~Hz}, f=143 \mathrm{~Hz}$, and $f \approx 1 \mathrm{kHz}$. Since in Fig. 15 the region of high coherence at high frequency has a relatively large frequency bandwidth, we have selected four modes to represent this source. We now describe each mode and examine its relationship to the frequencies at which high coherence between surface pressure and velocity are observed.

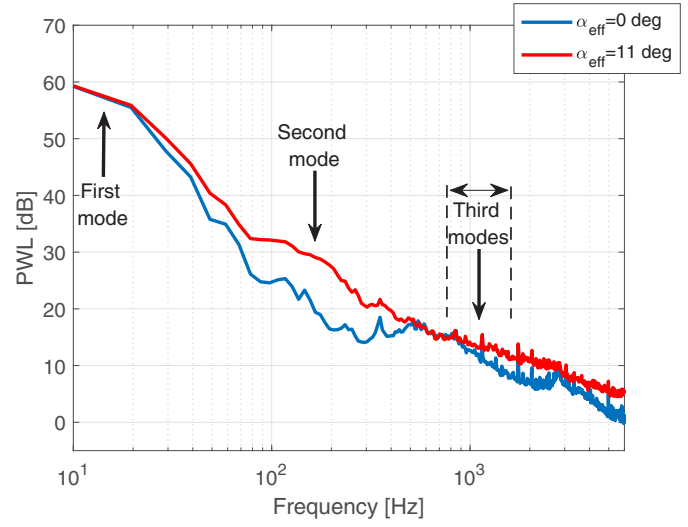

(a)

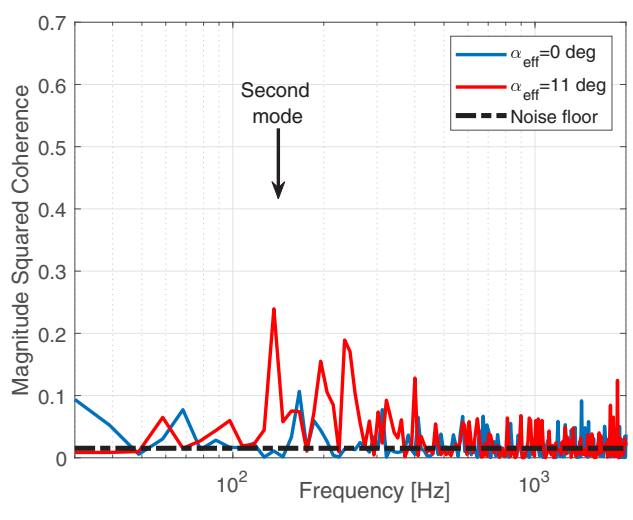

(b)

FIG. 19. Sound power level (left) and coherence between far-field noise and surface pressure on suction side (right) at $\alpha_{\text {eff }}=0^{\circ}$ and $\alpha_{\text {eff }}=11^{\circ}$ at $U_{\infty}=20 \mathrm{~m} / \mathrm{s}$. The dashed line in panel (b) represents the statistical noise floor. 


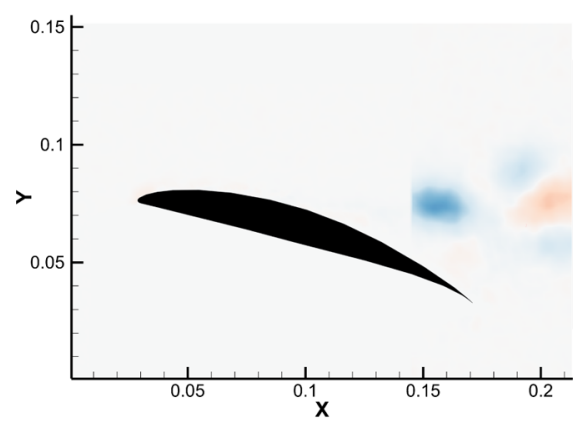

(a)

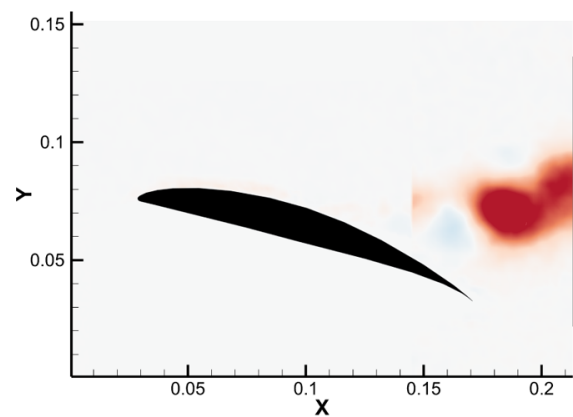

(c)

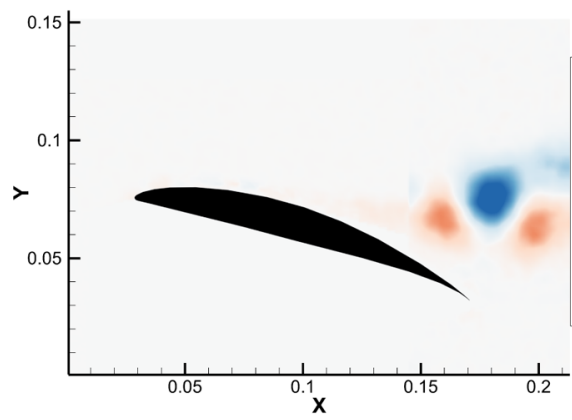

(e)

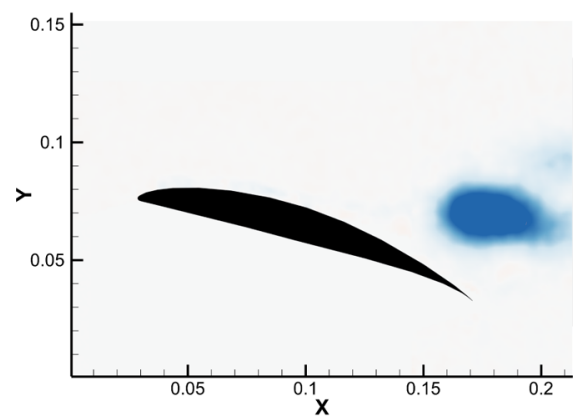

(g)

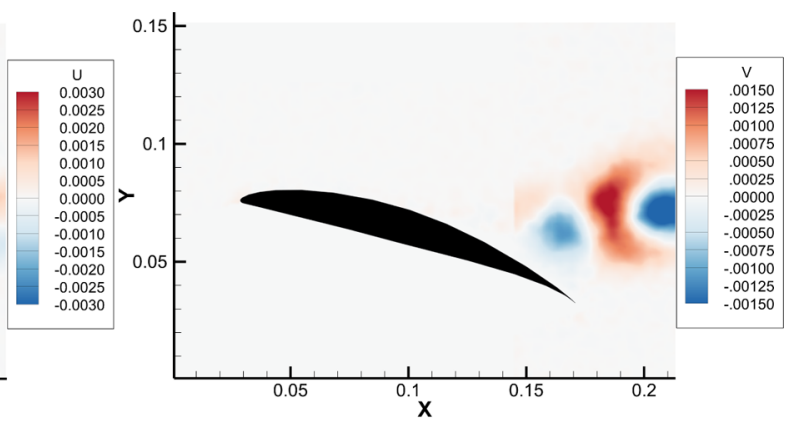

(b)
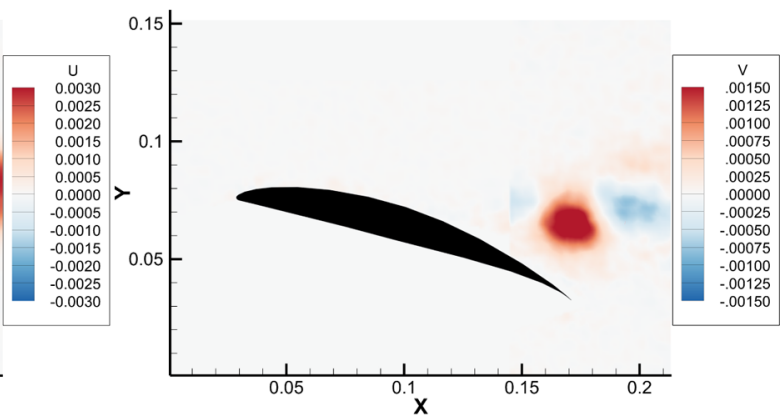

(d)

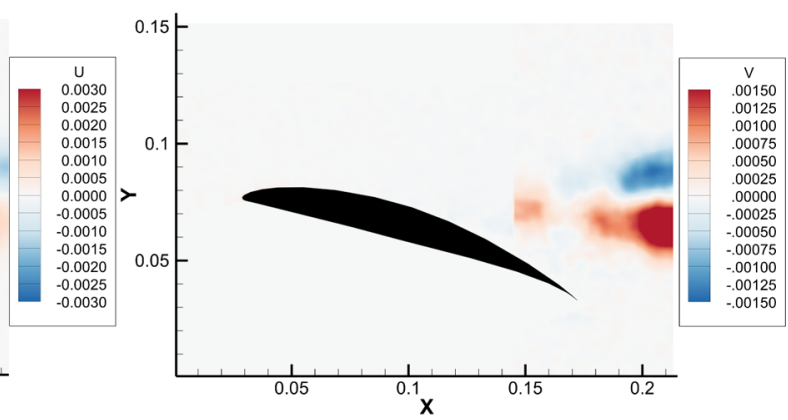

(f)

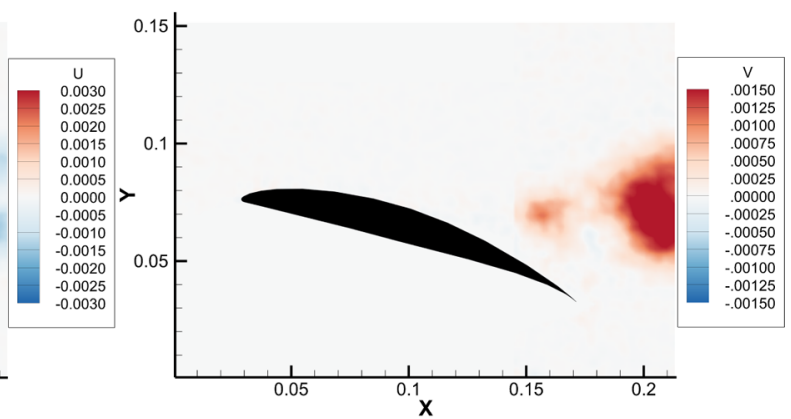

(h)

FIG. 20. High-order DMD modes: (left) streamwise $U$ velocity component; (right) vertical $V$ velocity component. (a) and (b) $f=884 \mathrm{~Hz}$, (c) and (d) $f=936 \mathrm{~Hz}$, (e) and (f) $f=1068 \mathrm{~Hz}$, and (g) and (h) $f=1155 \mathrm{~Hz}$. 


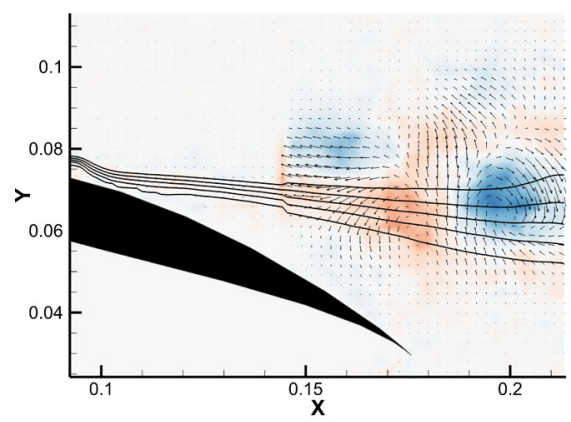

(a)

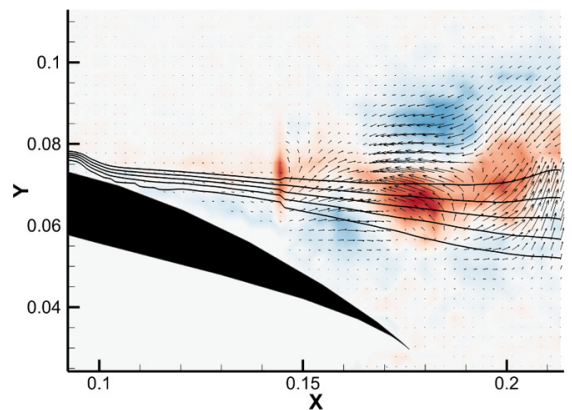

(c)

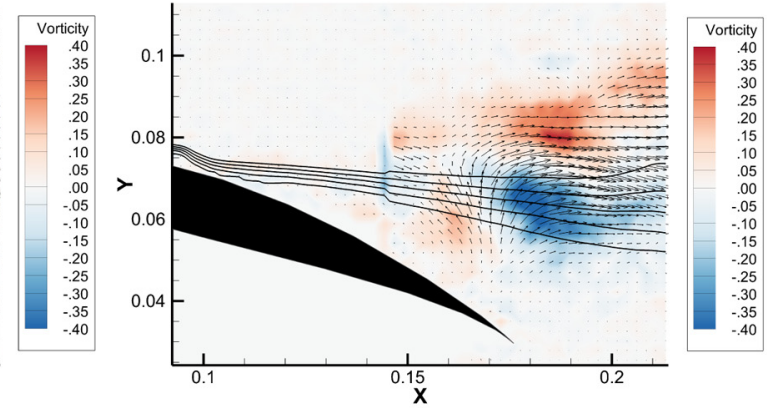

(b)
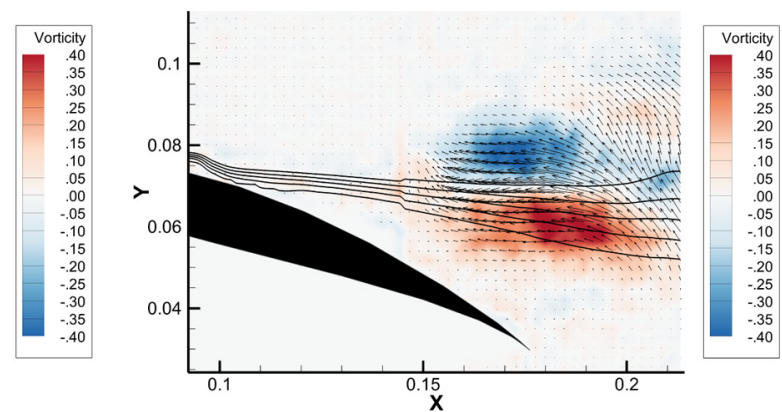

(d)

FIG. 21. High-order DMD modes: Zoomed vorticity contour and velocity vectors with overlapped contour levels of the shear layer: (a) $f=884 \mathrm{~Hz}$, (b) $f=936 \mathrm{~Hz}$, (c) $f=1068 \mathrm{~Hz}$, and (d) $f=1155 \mathrm{~Hz}$.

The first mode (indicated by the purple arrow in Fig. 16), having an associated frequency $f=$ $16 \mathrm{~Hz}$, has a relatively low decay rate and significant amplitude, corresponding to a low DA ratio. This mode is therefore persistent and contributes significantly to the reconstruction of the overall flow field. As discussed above, this frequency is consistent with the Strouhal number associated with flapping of the shear layer in conditions of incipient separation. The DMD modes of the streamwise and vertical velocity components associated with this frequency are shown in Figs. 17(a) and 17(b), respectively. They consist of regions of alternating higher and lower streamwise velocity above the shear layer, while the vertical velocity component depicts a recirculating flow across the shear itself. Figure 16(c) shows the contour of the instantaneous vorticity overlaid with the velocity vectors. It identifies areas of high negative and positive vorticity, possibly induced by the flapping shear layer, which occurs both within the shear and well above it.

The second DMD mode, indicated by the red arrow in the spectrum of Fig. 16, has a frequency of $146 \mathrm{~Hz}$, relatively low decay rate, and slightly lower velocity amplitude, corresponding to a midvalue DA ratio. As for the previous mode, this mode is also persistent. The streamwise and vertical velocity components of this mode are represented in Figs. 18(a) and 18(b). It can be seen to be characterized by a strong streamwise velocity component, but the vertical component above the shear layer is in the direction towards the shear while on the other side it is moving away from the shear layer. The resultant velocity vectors plotted in Fig. 18(c) give the appearance of flow converging axially as it moves downstream. Also shown in the figure is the spanwise vorticity, which can be seen to be rotating clockwise in the shear layer with induced countervorticity above the shear layer.

The frequency of this mode of $f=146 \mathrm{~Hz}$ can be seen in Fig. 19(a) to match the peak of the spectral hump in the far-field noise spectrum and the frequency of high coherence between the 
surface pressure and the far-field noise at $U_{\infty}=20 \mathrm{~m} / \mathrm{s}$, plotted in Fig. 19(b). It is therefore evident that this mode has an important role in the noise generation.

The coherence between streamwise velocity and surface pressure plotted in Fig. 15 shows a third region of relatively high coherence in the frequency range between $f \approx 0.8 \mathrm{kHz}$ and $f \approx 1.3 \mathrm{kHz}$. However, unlike the first two regions, it is confined to a localized $1 \mathrm{~cm}$ region just above the shear layer. The DMD spectrum in Fig. 16 shows that there are at least four modes ( $f=884,936,1068$, and $1155 \mathrm{~Hz}$ ) in this frequency band, all having a relatively low decay rate and middle to low amplitudes. The associated DA ratio is consequently low or middle/low. The flow fields associated with these four modes, which are depicted in Fig. 20 and 21, can be seen to differ in character. The first mode at $f=884 \mathrm{~Hz}$ in Fig. 20(a) indicates regions of alternating vorticity located inside the shear layer. The scale of these regions appear similar to the shear layer thickness of about $2 \mathrm{~cm}$. Coherent vorticity of this scale convecting at $U=20 \mathrm{~m} / \mathrm{s}$ will generate noise at a characteristic frequency $f \approx 20 / 0.02=1 \mathrm{kHz}$, which is entirely consistent with the modal frequency. The second mode with frequency $f=936 \mathrm{~Hz}$ in Fig. 20(b) shows evidence of fluid being ejected upwards and then convecting downstream with the flow. The third mode at $f=1068 \mathrm{~Hz}$ has a similar character as the first mode. Finally, the fourth mode at a frequency of $f=1155 \mathrm{~Hz}$ is characterized by two elongated regions of opposite sign vorticity within and above the shear layer.

\section{CONCLUSIONS}

In this paper the sound generated by an airfoil has been investigated experimentally over a wide range of angles of attack, including prestall and poststall conditions. Detailed hot wire and PIV measurements of the unsteady velocity around the airfoil were also made. In addition, static pressure measurements on both sides of the airfoil were also performed to assess where flow separation has occurred. The unsteady surface pressure and velocity throughout the boundary layer and shear layer were measured simultaneously and analysis applied to determine the regions in the flow that were coherent with the surface pressure near the trailing edge, which was found to be responsible for far-field radiation. Modal analysis on the PIV data was performed to extract the dominant flow characteristics at the frequencies of maximum coherence between surface pressure and velocity.

The study concentrates on a NACA65-(12)10 airfoil, but most of the findings can be generalized to different airfoils. Our aim was understanding in more detail the generation mechanism of the so-called separation/stall noise, being one of the possible self-noise sources of an airfoil. An understanding of this mechanism may lead to approaches to mitigate this noise source.

The conclusions from this work may be summarized as follows:

(1) Three possible mechanisms have been identified to explain the noise generation due to an airfoil close to stall. Flapping of the shear is a cause of very low-frequency sound, while the formation of coherent structures and instabilities in the shear layer has also been identified as possible noise sources for the separation/stall noise at low to middle frequencies. In all cases, these sources produce strong hydrodynamic pressure fluctuation close to the trailing edge, which then scatter into sound.

(2) The noise spectrum due to airfoil at varying angle of attack has been shown to fall into four categories. The first is at a low angle of attack where the boundary layer is attached. Here the radiated noise spectrum is relatively broadband. The second occurs at angles of attack where the flow has partially separated. In this case the noise spectrum is characterized by a relatively narrow peak whose peak frequency reduces as angle of attack increases. The third identifies the condition of maximum overall noise radiation and occurs at the angle of attack where the flow just becomes fully separated such that the point of separation has reached the leading edge. At this condition the noise spectrum is also broadband. Finally, the fourth region occurs at poststall angles of attack. Here the noise spectrum becomes increasingly broadband as angle of attack increases, but its overall level begins to reduce before increasing again due to a bluff body behavior. 
(3) Analysis has revealed the locations in the flow whose coherence between the unsteady surface pressure near the trailing edge, and the local velocity is maximum. The analysis was undertaken for the airfoil at $11^{\circ}$ angle of attack, corresponding to the second noise spectrum region defined above. These locations may be regarded as the sources of separation noise throughout the shear layer. Each of these sources occurs over a particular frequency range and spatial region. Low-frequency sources appear to be distributed over a large region above the shear layer. At higher frequencies, however, the sources appear more localized but still above the shear layer.

(4) The velocity modes at the frequencies of maximum coherence were investigated through modal analysis of the PIV velocity data. The mode associated with the frequency hump of the noise spectrum was identified and shown to be characterized by a region of negative spanwise vorticity located entirely within the shear layer and similar size region above the shear of positive vorticity. The modes associated with the lower and higher frequency components of the spectrum were also identified.

\section{ACKNOWLEDGMENT}

The authors would like to thank funders for the financial support from the EPSRC Research Grant No: EP/N020413/1 on the "Quiet Aerofoils of the Next Generation" at the University of Southampton.

[1] T. F. Brooks, D. S. Pope, and M. A. Marcolini, Airfoil self-noise and prediction, Technical Report No. NASA-RP-1218, NASA Langley Research Center, Hampton, VA, United States, 1989.

[2] R. A. Wallis, Wind tunnel studies of leading edge separation phenomena on a quarter scale model of the outer panel of the Handley Page "Victor" wing, with and without nose droop, Reports and Memoranda of the Aeronautical Research Council No. 3455 (The Stationery Office, London, 1965).

[3] H. Schlichting and K. Gersten, Boundary-Layer Theory (Springer, New York, 2016).

[4] R. Amiet, Noise due to turbulent flow past a trailing edge, J. Sound Vib. 47, 387 (1976).

[5] F. Bertagnolio, H. A. Madsen, A. Fischer, and C. Bak, A semi-empirical airfoil stall noise model based on surface pressure measurements, J. Sound Vib. 387, 127 (2017).

[6] M. Fink and D. Bailey, Airframe noise reduction studies and clean-airframe noise investigation, Final Technical Report No. NASA-CR-159311, United Technologies Research Center, East Hartford, United States, 1980.

[7] R. W. Paterson, R. K. Amiet, and C. L. Munch, Isolated airfoil-tip vortex interaction noise, J. Aircr. 12, 34 (1975).

[8] S. Moreau, M. Roger, and J. Christophe, Flow features and self-noise of airfoils near stall or in stall, AIAA paper 3198, 2009 (2009).

[9] C. Y. Schuele and K.-S. S. Rossignol, Trailing-edge noise modeling and validation for separated flow conditions, in 19th AIAA/CEAS Aeroacoustics Conference (AIAA, Reston, VA, 2013), p. 2008.

[10] A. Suryadi and M. Herr, Wall pressure spectra on a DU96-W-180 profile from low to pre-stall angles of attack, in 21st AIAA/CEAS Aeroacoustics Conference (AIAA, Reston, VA, 2015), p. 2688.

[11] S. Yarusevych, P. E. Sullivan, and J. G. Kawall, Coherent structures in an airfoil boundary layer and wake at low Reynolds numbers, Phys. Fluids 18, 044101 (2006).

[12] J. M. Lin and L. L. Pauley, Low-Reynolds-number separation on an airfoil, AIAA J. 34, 1570 (1996).

[13] S. Yarusevych, P. E. Sullivan, and J. G. Kawall, On vortex shedding from an airfoil in low-Reynoldsnumber flows, J. Fluid Mech. 632, 245 (2009).

[14] S. Burgmann and W. Schröder, Investigation of the vortex induced unsteadiness of a separation bubble via time-resolved and scanning PIV measurements, Exp. Fluids 45, 675 (2008).

[15] T. Berk, G. Lacagnina, C. Paruchuri, P. Joseph, and B. Ganapathisubramani, Simultaneous surface pressure and high-speed PIV measurements in stalled airfoil, in 19th International Symposium on 
Applications of Laser Techniques to Fluid Mechanics (Instituto Superior Tecnico, Lisbon, 2018), pp. 232-245.

[16] J. H. Watmuff, Evolution of a wave packet into vortex loops in a laminar separation bubble, J. Fluid Mech. 397, 119 (1999).

[17] P. J. Shapiro, The influence of sound upon laminar boundary layer instability, Acoustics and Vibration Laboratory Report No. 83458-83560, Massachusetts Institute of Technology, Cambridge, MA, 1977.

[18] K. Zaman, A. Bar-Sever, and S. Mangalam, Effect of acoustic excitation on the flow over a low-Re airfoil, J. Fluid Mech. 182, 127 (1987).

[19] K. Zaman, D. McKinzie, and C. Rumsey, A natural low-frequency oscillation of the flow over an airfoil near stalling conditions, J. Fluid Mech. 202, 403 (1989).

[20] I. H. Abbott and A. E. Von Doenhoff, Theory of Wing Sections, Including a Summary of Airfoil Data (Courier Corporation, New York, 1959).

[21] T. Chong, P. Joseph, and P. Davies, Design and performance of an open jet wind tunnel for aero-acoustic measurement, Appl. Acoustics 70, 605 (2009).

[22] T. Brooks, M. Marcolini, and D. Pope, Airfoil trailing edge flow measurements and comparison with theory, incorporating open wind tunnel corrections, in Ninth Aeroacoustics Conference (AIAA, Reston, VA, 1984), p. 2266.

[23] C. E. Willert and M. Gharib, Digital particle image velocimetry, Exp. Fluids 10, 181 (1991).

[24] M. Raffel, C. E. Willert, J. Kompenhans et al., Particle Image Velocimetry: A Practical Guide (Springer Science \& Business Media, New York, 2007).

[25] C. Cierpka, B. Lütke, and C. J. Kähler, Higher order multi-frame particle tracking velocimetry, Exp. Fluids 54, 1533 (2013).

[26] R. J. Adrian and J. Westerweel, Particle Image Velocimetry (Cambridge University Press, 2011).

[27] J. Soria, An investigation of the near wake of a circular cylinder using a video-based digital crosscorrelation particle image velocimetry technique, Exp. Therm. Fluid Sci. 12, 221 (1996).

[28] F. Scarano, Iterative image deformation methods in PIV, Meas. Sci. Technol. 13, R1 (2001).

[29] H. Huang, H. Fiedler, and J. Wang, Limitation and improvement of PIV, Exp. Fluids 15, 263 (1993).

[30] K. Jambunathan, X. Ju, B. Dobbins, and S. Ashforth-Frost, An improved cross correlation technique for particle image velocimetry, Meas. Sci. Technol. 6, 507 (1995).

[31] J. Nogueira, A. Lecuona, and P. Rodriguez, Local field correction PIV: On the increase of accuracy of digital PIV systems, Exp. Fluids 27, 107 (1999).

[32] J. Westerweel and F. Scarano, Universal outlier detection for PIV data, Exp. Fluids 39, 1096 (2005).

[33] M. Drela, Xfoil: An analysis and design system for low Reynolds number airfoils, in Low Reynolds Number Aerodynamics, edited by T. J. Mueller (Springer, 1989), pp. 1-12.

[34] I. Tani, Low-speed flows involving bubble separations, Prog. Aerospace Sci. 5, 70 (1964).

[35] J. D. Anderson Jr., Fundamentals of Aerodynamics (McGraw-Hill Education, New York, 2010).

[36] S. Narayanan, P. Chaitanya, S. Haeri, P. Joseph, J. Kim, and C. Polacsek, Airfoil noise reductions through leading edge serrations, Phys. Fluids 27, 025109 (2015).

[37] W. K. Blake, Mechanics of Flow-Induced Sound and Vibration, Volume 2: Complex Flow-Structure Interactions (Academic Press, Cambridge, Massachussets, 1986).

[38] R. Parchen, Progress report DRAW: A prediction scheme for trailing edge noise based on detailed boundary layer characteristics, Technical Report No. HAGRPT-980023, TNO Institute of Applied Physics, Delft, The Netherlands, 1998.

[39] O. Stalnov, P. Chaitanya, and P. F. Joseph, Towards a non-empirical trailing edge noise prediction model, J. Sound Vib. 372, 50 (2016).

[40] R. E. Sheldahl and P. C. Klimas, Aerodynamic characteristics of seven symmetrical airfoil sections through 180-degree angle of attack for use in aerodynamic analysis of vertical axis wind turbines, Technical Report No. SAND-80-2114, Sandia National Labs, Albuquerque, USA, 1981.

[41] N. Gregory and C. O'Reilly, Low-Speed Aerodynamic Characteristics of NACA 0012 Aerofoil Section, Including the Effects of Upper-Surface Roughness Simulating Hoar Frost (HM Stationery Office, London, 1973). 
[42] D. E. Gault, A correlation of low-speed, airfoil-section stalling characteristics with Reynolds number and airfoil geometry, NASA Technical Note 3963, National Advisory Committee for Aeronautics, Ames Aeronautical Lab., CA, United States, 1957.

[43] M. Brendel and T. J. Mueller, Boundary-layer measurements on an airfoil at low Reynolds numbers, J. Aircr. 25, 612 (1988).

[44] P. Schmid and J. Sesterhenn, Dynamic Mode Decomposition of numerical and experimental data, in 61 st Annual Meeting of the APS Division of Fluid Dynamics (APS, New York, 2008), p. MR.007.

[45] P. J. Schmid, Dynamic mode decomposition of numerical and experimental data, J. Fluid Mech. 656, 5 (2010).

[46] B. O. Koopman, Hamiltonian systems and transformation in Hilbert space, Proc. Natl. Acad. Sci. U.S.A. 17, 315 (1931).

[47] S. Le Clainche and J. M. Vega, Higher order dynamic mode decomposition, SIAM J. Appl. Dyn. Syst. 16, 882 (2017).

[48] S. Le Clainche, J. M. Vega, and J. Soria, Higher order dynamic mode decomposition of noisy experimental data: The flow structure of a zero-net-mass-flux jet, Exp. Therm. Fluid Sci. 88, 336 (2017). 